\title{
Generalization and Exact Deformations of Quantum Groups
}

\author{
Christian FrøNSDAL*
}

\begin{abstract}
A large family of "standard" coboundary Hopf algebras is investigated. The existence of a universal R-matrix is demonstrated for the case when the parameters are in general position. Algebraic surfaces in parameter space are characterized by the appearance of certain ideals; in this case the universal R-matrix exists on the associated algebraic quotient. In special cases the quotient is a "standard" quantum group; all familiar quantum groups including twisted ones are obtained in this way. In other special cases one finds new types of coboundary bi-algebras.

The "standard" universal R-matrix is shown to be the unique solution of a very simple, linear recursion relation. The classical limit is obtained in the case of quantized Kac-Moody algebras of finite and affine type.

Returning to the general case, we study deformations of the standard R-matrix and the associated Hopf algebras. A preliminary investigation of the first order deformations uncovers a class of deformations that incompasses the quantization of all Kac-Moody algebras of finite and affine type. The corresponding exact deformations are described as generalized twists, $R_{\varepsilon}=\left(F^{t}\right)^{-1} R F$, where $R$ is the standard R-matrix and the cocycle $F$ (a power series in the deformation parameter $\epsilon$ ) is the solution of a linear recursion relation of the same type as that which determines $R$. Included here is the universal R-matrix for the elliptic quantum groups associated with $s l(n)$, a big surprise!

Specializing again, to the case of quantized Kac-Moody algebras, and taking the classical limit of these esoteric quantum groups, one re-discovers all the trigonometric and elliptic r-matrices of Belavin and Drinfeld. The formulas obtained here are easier to use than the original ones, and the structure of the space of classical $r$-matrices is more transparent. The r-matrices obtained here are more general in that they are defined on the full Kac-Moody algebras, the central extensions of the loop groups.
\end{abstract}

\section{TABLE}

§1. Introduction

§2. Standard Universal R-matrices

§. Differential Algebras

§. Differential Complexes

Communicated by T. Miwa, February 6, 1996. Revised May 15, 1996.

1991 Mathematics Subject Classification(s): Primary, 16W30, Secondary, 14A22 and 14M12.

* Department of Physics and Astronomy, University of California, Los Angeles CA 90095-1547, U.S.A. 
§5. Integrability of Eq. (2.14)

§6. Completion of the Proof of Theorem 2

$\S 7 . \quad$ Obstructions and Generalized Serre Relations

§. The Standard Classical r-matrix for Simple Lie Algebras

§. The Standard Classical r-matrix for Untwisted Loop Algebras

$\S 10$. The Standard Classical r-matrix for Twisted Loop Algebras

$\S 11$. Including the Central Extension

\section{DEFORMATIONS}

§12. First order Deformations

$\S 13$. First order Deformations of Type $e_{\sigma} \otimes e_{-\rho}$ and the Classical Limit

$\S 14$. Hopf Structure

$\S 15$. Exact Deformations of Standard, Generalized Quantum Groups

\$16. Esoteric r-matrices

$\S 18$. Universal Elliptic R-and r-matrices

Acknowledgements

References

\section{§1. Introduction.}

Quantum groups sprouted in that fertile soil where mathematics overlaps with physics. The mathematics of quantum groups is exciting, and the applications to physical modelling are legion. It is the more surprising that some aspects of the structure of quantum groups remain to be explored; this is especially true of those aspects that bear upon the problem of classification. The quantum groups that have so far found employment in physics are very special (characterized by a single "deformation" parameter $q$ ). It is true that these applications are susceptible to some generalization, by the process of "Cartan twisting"; by this we mean the type of twisting that was used by Reshetikhin [R] to construct the multiparameter quantum groups, in which a quantum R-matrix $R$ is replaced by $\widetilde{R}=\left(F^{t}\right)^{-1} R F$, with $F$ in the Cartan subalgebra. Unfortunately it is easy to receive the impression that twisting is a gauge transformation that relates equivalent structures. The fact that Cartan-twisted or multiparameter quantum groups differ qualitatively among themselves becomes evident when one investigates their rigidity to deformation. Deformation theory is a means of attacking the classification problem; at the same time it offers a wider horizon against which to view the whole subject. The new quantum groups discovered this way (the deformations of the twisted ones) are dramatically different; the physical applications should be of a novel kind.

Let $g$ be a simple Lie algebra over $C$. A structure of coboundary Lie bialgebra on $\mathfrak{g}$ is determined by a "classical" r-matrix; an element $r \in \mathfrak{g} \otimes \mathfrak{g}$ that 
satisfies the classical Yang-Baxter relation

$$
\left[r_{12}, r_{13}+r_{23}\right]+\left[r_{13}, r_{23}\right]=0
$$

as well as the symmetry condition

$$
r+r^{t}=\hat{K}
$$

where $\hat{K}$ is the Killing form of $\mathfrak{g}$. The classification of r-matrices of simple complex Lie algebras (finite and affine) was accomplished by Belavin and Drinfeld [BD].

It is widely believed that there corresponds, to each such r-matrix, via a process of "quantization," a unique quantum group [D2]. Somewhat more precisely, one expects that there exists a Hopf algebra deformation $\tilde{U}(\mathrm{~g})$ of $U(\mathrm{~g})$, and an element $R \in \tilde{U}(\mathrm{~g}) \otimes \tilde{U}(\mathrm{~g})$, such that $\Delta R=R \Delta^{\prime}$, where $\Delta$ is the coproduct of $\tilde{U}(\mathrm{~g})$ and $\Delta^{\prime}$ is the opposite coproduct, satisfying the (quantum) Yang-Baxter relation

$$
R_{12} R_{13} R_{23}=R_{23} R_{13} R_{12}
$$

such that $r$ can be recovered by an expansion of $R$ with respect to a parameter $\hbar$ :

$$
R=1+\hbar r+o\left(\hbar^{2}\right)
$$

Till now, this program had been realized for $\mathrm{r}$-matrices of a class that we call "standard".

Definition 1.1. Let $g$ be a simple, complex Lie algebra, $\mathfrak{g}^{0}$ a Cartan subalgebra and $\Delta^{+}$a set of positive roots. A (constant) standard r-matrix for $\mathrm{g}$ has the expression

$$
r=r_{0}+\sum_{\alpha \in \Delta^{+}} E_{-\alpha} \otimes E_{\alpha}
$$

Here $r_{0} \in \mathfrak{g}^{0} \otimes \mathfrak{g}^{0}$ is restricted by (1.2). An affine r-matrix (non-constant, with spectral parameter) is of standard type if it commutes with the Cartan subalgebra.

The (universal) R-matrix that corresponds to a standard r-matrix is known. Explicit formulas are of two types: in terms of Serre generators, or in terms Lie generators. An explicit formula in terms of Lie generators has 
been given for the simplest choice of $r_{0}$ in [KR]. An expression for $R$ in terms of Drinfeld-Serre generators $[\mathrm{D} 1][\mathrm{FR}][\mathrm{LS}][\mathrm{Ro}][\mathrm{T}]$ seems more fundamental (especially so in the affine case),

$$
R=R^{0}\left(1+\sum_{\alpha} e_{-\alpha} \otimes e_{\alpha}+\cdots\right)
$$

Here $\left\{H_{a}, e_{\alpha}, e_{-\alpha}\right\}$ are Chevalley-Drinfeld generators associated with a Cartan subalgebra and simple roots, $R^{0}$ involves only the $H_{a}$ 's. An R-matrix of this form will be called standard; a precise definition (in a more general context) will be given in Section 2, Definition 2.2. The relationship between (1.5) and (1.6) is examined in Sections 8 and 16. An explicit formula for the coefficients in (1.6) is in (5.9).

The R-matrices associated with the multiparameter quantum groups discovered by Reshetikhin [R] and others $[\mathrm{Sc}][\mathrm{Su}]$ are thus all included in the rubrique "standard". The principal characteristic of a standard R-matrix is that it "commutes with Cartan":

$$
\left[H_{a} \otimes 1+1 \otimes H_{a}, R\right]=0 .
$$

Until now, non-standard R-matrices were known only in the fundamental representation [CG][FG1].

The aim of this work is to use deformation theory to discover the so far unknown quantum groups that are alleged to be associated with non-standard r-matrices. This seems a reasonable approach because (i) non-standard $r$-matrices can be viewed, and effectively calculated $[F]$, as deformations of standard r-matrices and (ii) the largest family of non-standard quantum groups known so far was found by applying deformation theory to certain standard $\mathrm{R}$-matrices in the fundamental representation [FG2].

Progress achieved in the present paper is due, in the first place, to the idea of focusing on the representation (1.6) of the standard universal R-matrix, and in the second place to the discovery of a differential complex associated with the Yang-Baxter relation: the study of (1.6) turned out to be unexpectedly rewarding.

The existence of a universal R-matrix in the form (1.6), for quantized Lie algebras and for Kac-Moody algebras, was known [D1][FR][T]. But it turns out that the representation (1.6) for an R-matrix that satisfies the Yang-Baxter equation makes sense in a context that is much wider than quantized Kac-Moody algebras. We present a proof of the existence of an R-matrix of the form (1.6) that covers a wider category of bialgebras. The proof is constructive 
and provides usefull insight into the structure of these bialgebras (actually Hopf algebras). It exploits a direct connection between the Yang-Baxter equation and a certain differential complex, and it reduces the calculation of $R$ to the solution of a linear recursion relation.

We introduce (Definition 2.1) an algebra $\mathscr{A}$ with generators $\left\{H_{a}, e_{\alpha}, e_{-\alpha}\right\}$ that satisfy certain relations, including the following (see also Eq. (1.7b) below):

$$
\left[H_{a}, H_{b}\right]=0, \quad\left[H_{a}, e_{ \pm \alpha}\right]= \pm H_{a}(\alpha) e_{ \pm \alpha}, \quad H_{a}(\alpha) \in C .
$$

We define a standard R-matrix on $\mathscr{A}$ - Definition 2.2 - as a formal series of the form

$$
R=\exp \left(\varphi^{a b} H_{a} \otimes H_{b}\right)\left(1+e_{-\alpha} \otimes e_{\alpha}+\sum_{k=2}^{\infty} t_{(\alpha)}^{\left(\alpha^{\prime}\right)} e_{-\alpha_{1}} \cdots e_{-\alpha_{k}} \otimes e_{\alpha_{1}^{\prime}} \cdots e_{\alpha_{k}}\right)
$$

with parameters $\varphi^{a b} \in C$, fixed, and determine the coefficients $t_{(\alpha)}^{\left(\alpha^{\prime}\right)} \in C$ so that the Yang-Baxter relation (1.3) is satisfied. One finds that this requires additional relations, namely

$$
\left[e_{\alpha}, e_{-\beta}\right]=\delta_{\alpha}^{\beta}\left(e^{\varphi(\alpha \cdot)}-e^{-\varphi(\cdot \alpha)}\right)
$$

with $\varphi(\alpha, \cdot)=\varphi^{a b} H_{a}(\alpha) H_{b}, \varphi(\cdot, \alpha)=\varphi^{a b} H_{a} H_{b}(\alpha)$. These relations are therefore included in the definition of the algebra $\mathscr{A}$. Generically, with the parameters in general position, no further relations are required.

The generators $H_{a}$ of $\mathscr{A}$ generate an Abelian subalgebra $\mathscr{A}^{0}$ that may be called the Cartan subalgebra. A key point is to refrain from introducing, a priori, any (generalized) Serre relations among the Chevalley-Drinfeld generators $e_{\alpha}$, or among the $e_{-\alpha}$. The algebras of ultimate interest are obtained subsequently, by identifying an appropriate ideal $I \subset \mathscr{A}$ that intersects $\mathscr{A}^{0}$ trivially, and passing to the quotient $\mathscr{A}^{\prime}=\mathscr{A} / I$. This is the strategy of Chevalley [C], fully exploited in the theory of Kac-Moody algebras [K][Mo]; here it is applied to "generalized quantum groups."

This point of view allows a significant generalization. We study free differential algebras in general, then attempt to classify the ideals. To each ideal there corresponds a quotient algebra on which a coboundary Hopf algebra (with its standard R-matrix) can be constructed. Quantized KacMoody algebras, characterized by Serre-Drinfeld ideals, form a special case.

The first result is Theorem 2. It asserts that the Yang-Baxter relation for the standard R-matrix on $\mathscr{A}$ is equivalent to a simple, linear recursion relation for the coefficients $t_{(\alpha)}^{\left(\alpha^{\prime}\right)}$. This result is of great help in the subsequent 
calculations.

The integrability of this recursion relation, Eq. (2.14), is related to the first cohomology group on quantum planes. Generically, all one-forms are exact, whence the second result that, when the parameters of $\mathscr{A}$ are in general position, there exists a unique set of coefficients $t_{(\alpha)}^{\left(\alpha^{\prime}\right)}$ such that the standard R-matrix satisfies the Yang-Baxter relation.

Obstructions to the solution of the recursion relation (2.14), and thus to the Yang-Baxter relation on $\mathscr{A}$, exist on certain hyper-surfaces in the space of parameters of $\mathscr{A}$, they are detected by the presence of "constants". A Serre relation is a special type of constant. Constants are studied in a general context in $\$ \S 3$ and 4; their complete classification is an open, but probably not unsolvable problem. Its solution would shed light on the structure of ideals in quantized Kac-Moody algebras and reduce the theorem of Gabber and $\mathrm{Kac}[\mathrm{GK}]$ to a corollary. The relevance of this discussion to the Yang-Baxter relation is demonstrated in $\S 5$, and the proof of Theorem 2 is completed in $\S 6$.

The study of the obstructions is taken up again in $\$ 7$. The third main result is Theorem 7: the obstructions (that is, the constants) generate an ideal $I \subset \mathscr{A}$, and a unique standard R-matrix, satisfying Yang-Baxter, exists on $\mathscr{A} / I$.

Next, we specialize to quantized Kac-Moody algebras and calculate the classical limit, that is, the classical r-matrix associated with the standard R-matrix. The result was of course known, but without a precise determination of $R$ it is not possible to evaluate the limit directly. A further complication is that all the coefficients become singular. But the recursion relation (2.14) guarantees the existence of the limit and provides an efficient method for evaluating it. See $\$ \S 9,10$ and $16,17$.

Quantized Kac-Moody algebras are characterized by the property that, for each pair $(\alpha, \beta)$, there is a positive integer $k=k_{\alpha \beta}$ such that the following relation holds

$$
e^{\varphi(\alpha, \beta)+\varphi(\beta, \alpha)+(k-1) \varphi(\alpha, \alpha)}=1
$$

In this case the ideal $I$ is generated by the Serre relations

$$
0=\sum_{m=0}^{k} Q_{m}^{k}\left(e_{\alpha}\right)^{m} e_{\beta}\left(e_{\alpha}\right)^{k-m},
$$

with coefficients 


$$
Q_{m}^{k}=(-)^{m} e^{m \varphi(\alpha, \beta)} q^{m(m-1) / 2}\left(\begin{array}{l}
k \\
m
\end{array}\right)_{q}, \quad q:=e^{\varphi(\alpha, \alpha)}
$$

We suppose Card $N$ and Card $M$ finite and interpret $A=1-k$ as the generalized Cartan matrix of a Kac-Moody algebra.

The classical $r$-matrix associated with $R$ is defined after rescaling of the generators as the coefficient of $\hbar$ in the expansion $R=1+\hbar r+o\left(\hbar^{2}\right)$; it satisfies the classical Yang-Baxter relation. (Note that $r+r^{t} \neq 0$; the antisymmetric part of $r$ satisfies the modified classical Yang-Baxter relation). We calculate this classical r-matrix, dealing separately with the following cases: Kac-Moody algebras of finite type in $\S 8$; unextended loop algebras (untwisted and twisted) in $\S \S 9$ and 10; the full Kac-Moody algebras in $\S 11$.

\section{DEFORMATIONS}

The rest of the paper is a study of the deformations of the standard R-matrix, satisfying the Yang-Baxter relation, but in the wider context of the bialgebras $\mathscr{A}$ and $\mathscr{A}^{\prime}=\mathscr{A} / I$ described above. We set

$$
R_{\epsilon}=R+\epsilon R_{1}+o\left(\epsilon^{2}\right)
$$

and suppose that $R_{1}$ is driven by a term of the type

$$
S e_{-\rho} \otimes e_{\sigma}+S^{\prime} e_{\sigma} \otimes e_{-\rho}, \quad S, S^{\prime} \in \mathscr{A}^{\prime 0} .
$$

Such deformations exist under certain conditions on the parameters; then $S$ and $S^{\prime}$ and the remaining terms in $R_{\epsilon}$ (a formal power series in $\epsilon$ with constant term $R$ ) are determined by the Yang-Baxter relation.

We begin by calculating a class of first order deformations of the standard R-matrix on $\mathscr{A} / I$ for any ideal of obstructions $I \subset \mathscr{A}$. This is our fourth result, Theorem 13.1. The main difficulty is that the problem is not well posed, for we have been unable to discover a category that is both natural and convenient in which to calculate all deformations. We limit our study to a class of deformations. The good news is Theorem 13.2: when we specialize to the case of simple quantum groups, then we obtain quantizations of all simple Lie bialgebras of Belavin and Drinfeld, so far to first order in the deformation parameter.

In $\S 14$ we define the coproduct, counit and antipode that turn all these algebras into Hopf algebras. This completes the investigation of first order deformations. The results provide inspiration for construction of exact 
deformations.

An exact formula (to all orders in $\epsilon$ ) in closed form for $R_{\epsilon}$ is obtained for the case of elementary deformations, when $R_{1}$ is a single term of the type (1.10). In the general case of compound deformations, when (1.10) is replaced by a sum of terms of the same type, we obtain exact deformations in the form of a generalized twist. ( $\$ 15$.)

Let $R$ be the R-matrix of a coboundary Hopf algebra $\mathscr{A}^{\prime}$, and $F \in \mathscr{A}^{\prime} \otimes \mathscr{A}^{\prime}$, invertible.

Then

$$
\tilde{R}:=\left(F^{t}\right)^{-1} R F
$$

satisfies the Yang-Baxter relation if $F$ satisfies the cocycle condition [G]

$$
\left(\left(1 \otimes \Delta_{21}\right) F\right) F_{12}=\left(\left(\Delta_{13} \otimes 1\right) F\right) F_{31} .
$$

(See Theorem 15.1 for the complete statement.) Though it is not quite germaine to our discussion, it may be worth while to point out that, if $R$ is unitary, then so is $\tilde{R}$; the formula (1.11) therefore yields a family of (mostly) new unitary R-matrices.

Applying this to our context, we find that the relation (1.12) is equivalent to a simple, linear recursion relation that can be reduced to the same form as the recursion relation that determines the coefficients in the expansion of $R$. It has a unique solution that can be expressed directly in terms of these coefficients. Just as in the standard case, this leads to a simple equation for the classical r-matrix, from which the latter is determined to all orders.

In $\S 16$ we specialize to the case of deformed quantized, affine Kac-Moody algebras and take the classical limit, to recover the esoteric, affine r-matrices of the simple Lie algebras, with their central extensions. The result agrees with that of Belavin and Drinfeld, except that they did not include the central extension. The formulas obtained in this paper are more transparent and simpler to use.

Finally, in $\$ 17$, we deal with a very special case, to discover that the elliptic r-matrices of $s l(N)$ also arise as the classical limit of certain deformed quantum groups. The universal elliptic R-matrix is expressed as an infinite product. It is shown, in the particular case of the elliptic R-matrix for sl(2) in the fundamental representation, that this infinite product is both convergent and of practical utility; it reduces to the representation of elliptic functions in terms of infinite products, and the result is in perfect agreement with Baxter [B]. 


\section{§2. Standard Universal R-matrices.}

The universal R-matrix of a standard or twisted quantum group has the form

$$
\begin{aligned}
R= & \exp \left(\varphi^{a b} H_{a} \otimes H_{b}\right) \\
& \times\left(1+t_{\alpha}\left(e_{-\alpha} \otimes e_{\alpha}\right)+t_{\alpha \beta}\left(e_{-\alpha} e_{-\beta} \otimes e_{\alpha} e_{\beta}\right)+t_{\alpha \beta}^{\prime}\left(e_{-\alpha} e_{-\beta} \otimes e_{\beta} e_{\alpha}\right)+\cdots\right) .
\end{aligned}
$$

The $H_{a}$ are generators of the Cartan subalgebra, the $e_{\alpha}$ are generators associated with simple roots, $\varphi^{a b}, t_{\alpha}, t_{\alpha \beta}, t_{\alpha \beta}^{\prime}, \cdots$ are in the field; the unwritten terms are monomials in the $e_{\alpha}$ and $e_{-\alpha}$.

More generally, consider the expression (2.1) in the more general case when $H_{a}, e_{ \pm \alpha}$ generate an associative algebra with the following relations.

Definition 2.1. Let $M, N$ be two countable sets, $\varphi, \psi$ two maps,

$$
\begin{aligned}
\varphi: M \times M \rightarrow C, & a, b \mapsto \varphi^{a b}, \\
\psi: M \times N \rightarrow C \quad & a, \beta \mapsto H_{a}(\beta) .
\end{aligned}
$$

Let $\mathscr{A}$ or $\mathscr{A}(\varphi, \psi)$ be the universal, associative, unital algebra over $C$ with generators $\left\{H_{a}\right\} a \in M,\left\{e_{ \pm \alpha}\right\} \alpha \in N$, and relations

$$
\begin{aligned}
& {\left[H_{a}, H_{b}\right]=0, \quad\left[H_{a}, e_{ \pm \beta}\right]= \pm H_{a}(\beta) e_{ \pm \beta},} \\
& {\left[e_{\alpha}, e_{-\beta}\right]=\delta_{\alpha}^{\beta}\left(e^{\varphi(\alpha \cdot)}-e^{-\varphi(\cdot \cdot \alpha)}\right)}
\end{aligned}
$$

with $\varphi(\alpha, \cdot)=\varphi^{a b} H_{a}(\alpha) H_{b}, \varphi(\cdot, \alpha)=\varphi^{a b} H_{a} H_{b}(\alpha)$ and $e^{\varphi(\alpha \cdot)+\varphi(\cdot \alpha)} \neq 1, \alpha \in N$.

The last condition on the parameters is included in order to avoid having to make some rather trivial exceptions.

The free subalgebra generated by $\left\{e_{\alpha}\right\} \alpha \in N$ (resp. $\left\{e_{-\alpha}\right\} \alpha \in N$ ) will be denoted $\mathscr{A}^{+}$(resp. $\mathscr{A}^{-}$); these subalgebras are $Z$-graded, the generators having grade 1. The subalgebra generated by $\left\{H_{a}\right\} a \in M$ is denoted $\mathscr{A}^{0}$. If necessary we may assume that $M$ is finite.

Definition 2.2. A standard R-matrix is a formal series of the form

$$
R=\exp \left(\varphi^{a b} H_{a} \otimes H_{b}\right)\left(1+e_{-\alpha} \otimes e_{\alpha}+\sum_{k=2}^{\infty} t_{\alpha_{1} \cdots \alpha_{k}}^{\alpha_{1}^{\prime} \cdots \alpha_{k}} e_{-\alpha_{1}} \cdots e_{-\alpha_{k}} \otimes e_{\alpha_{1}^{\prime}} \cdots e_{\alpha_{k}}\right)
$$

In this formula, and in others to follow, summation over repeated indices is 
implied.

For fixed $(\alpha)=\alpha_{1}, \cdots, \alpha_{k}$ the sum over $\left(\alpha^{\prime}\right)$ runs over the permutations of $(\alpha)$. The coefficients $t_{(\alpha)}^{\left(\alpha^{\prime}\right)}$ are in $\mathbb{C}$.

The special property associated with the qualification "standard" is that " $R$ commutes with Cartan"; indeed $\left[R, H_{a} \otimes 1+1 \otimes H_{a}\right]=0, a \in M$.

We shall determine under what conditions on the parameters $\varphi^{a b}, H_{a}(\beta)$ of $\mathscr{A}$, and for what values of the coefficients $t_{(\alpha)}^{\left(\alpha^{\prime}\right)}$, the R-matrix (2.5) satisfies the Yang-Baxter relation

$$
Y B:=R_{12} R_{13} R_{23}-R_{23} R_{13} R_{12}=0 .
$$

This expression is a formal series in which each term has the form $\psi_{1} \otimes \psi_{2} \otimes \psi_{3} \in \mathscr{A} \otimes \mathscr{A} \otimes \mathscr{A}$. We assign a double grading as follows. First extend the grading of $\mathscr{A}^{+}$to the subalgebra of $\mathscr{A}$ that is generated by $\left\{H_{a}\right\} a \in M$ and $\left\{e_{\alpha}\right\} \alpha \in N$, by assigning grade zero to $H_{a}$, and similarly for $\mathscr{A}^{-}$. Then $\psi_{1}$ and $\psi_{3}$ (but not $\psi_{2}$ ) belong to graded subalgebras of $\mathscr{A}$. If $\psi_{1}$ and $\psi_{3}$ have grades $l$ and $n$, respectively, then define

$$
\operatorname{grade}\left(\psi_{1} \otimes \psi_{2} \otimes \psi_{3}\right)=(l, n) \text {. }
$$

To give a precise meaning to (2.6) we first declare that we mean for this relation to hold for each grade $(l, n)$ separately. This is not enough, for the number of terms contributing to each grade is infinite in general. The appearance of exponentials in the $H_{a}$ can be dealt with in the same way as in the case of simple quantum groups [TV]. If the sets $M, N$ are infinite, then all results are basis dependent. Eq. (2.6) means that $Y B$, projected on any finite subalgebra of $\mathscr{A}$, vanishes on each grade; the statement thus involves only finite sums. The analysis of (2.6) will be organized by ascending grades.

Remarks. (i) It is an immediate consequence of (2.6), in grade (1.1), that

$$
\left[e_{\alpha}, e-\beta\right]=\delta_{\alpha}^{\beta}\left(e^{\varphi(\alpha, \cdot)}-e^{-\varphi(\cdot, \alpha)}\right) .
$$

This relation was therefore included in the definition of the algebra $\mathscr{A}$. (ii) No relations of the Serre type have been imposed; in fact no relations whatever on the subalgebras $\mathscr{A}^{+}$and $\mathscr{A}^{-}$, they are freely generated by the $e_{\alpha}$ and by the $e_{-\alpha}$, respectively. The contextual meaning of such relations, including relations of the Serre type, will be discussed in Sections 3-5 and especially in Section 7.

Before stating the main result, it will be convenient to show the direct 
evaluation of $Y B$ up to grade (2.2). We expand

$$
R^{0}:=\exp \left(\varphi^{a b} H_{a} \otimes H_{b}\right)=R^{i} \otimes R_{i},
$$

sums over $a, b, i$ implied. Then

$$
e_{-\alpha} R^{i} \otimes R_{i}=R^{i} e_{-\alpha} \otimes e^{\varphi(\alpha, \cdot)} R_{i} .
$$

Grade $(1,1)$. The contributions to $R_{12} R_{13} R_{23}$ are of two kinds:

$$
\begin{aligned}
& R^{i} R^{j} e_{-\alpha} \otimes R_{i} R^{k} \otimes R_{j} e_{\alpha} R_{k}, \\
& R^{i} e_{-\alpha} R^{j} \otimes R_{i} e_{\alpha} R^{k} e_{-\beta} \otimes R_{j} R_{k} e_{\beta} .
\end{aligned}
$$

Cancellation in $Y B$ is equivalent to Eq. (2.4).

Grade $(1,2)$. The contributions to $R_{12} R_{13} R_{23}$ are

$$
\begin{aligned}
& R^{i} R^{j} e_{-\beta} \otimes R_{i} R^{k} e_{-\alpha} \otimes R_{j} e_{\beta} R_{k} e_{\alpha}, \\
& t_{\alpha \beta^{\prime} \beta^{\prime}} R^{i} e_{-\gamma} R^{j} \otimes R_{i} e_{\gamma} R^{k} e_{-\alpha} e_{-\beta} \otimes R_{j} R_{k} e_{\alpha^{\prime}} e_{\beta^{\prime}} .
\end{aligned}
$$

Cancellation in $Y B$ requires that

$$
\begin{aligned}
& t_{\alpha \beta}^{\alpha \beta}=\left(1-e^{-\varphi(\alpha, \beta)-\varphi(\beta, \alpha)}\right)^{-1}, \\
& t_{\alpha \beta}^{\beta \alpha}=-e^{-\varphi(\beta, \alpha)} t_{\alpha \beta}^{\alpha \beta}, \quad \alpha \neq \beta, \\
& t_{\alpha \alpha}^{\alpha \alpha}=\left(1+e^{-\varphi(\alpha, \alpha)}\right)^{-1} .
\end{aligned}
$$

These conditions are necessary and sufficient that the standard R-matrix satisfy (2.6) up to grade $(2,2)$.

The obstructions to the existence of coefficients $t_{(\alpha)}^{\left(\alpha^{\prime}\right)}$ such that $Y B=0$ up to grade $(2,2)$ are therefore as follows:

$$
\begin{aligned}
& 1+e^{-\varphi(\alpha, \alpha)}=0 \text { for some } \alpha \in N, \\
& 1+e^{-\varphi(\alpha, \beta)-\varphi(\beta . \alpha)}=0 \text { for some pair } \alpha \neq \beta .
\end{aligned}
$$

They are typical of obstructions encountered at all grades.

Let

$$
t_{\alpha_{1} \cdots \alpha_{l}}:=t_{\alpha_{1} \cdots \alpha_{l}}^{\alpha_{1}^{\prime} \cdots a_{\alpha_{1}}} e_{1} \cdots e_{\alpha_{l}}
$$

Theorem 2. The standard R-matrix (2.5), on $\mathscr{A}$, satisfies the Yang-Baxter relation (2.6) if and only if the coefficients $t_{(\alpha)}^{\left(\alpha^{\prime}\right)}$ satisfy the following recursion 
relation

$$
\left[t_{\alpha_{1} \cdots \alpha_{l}}, e-\gamma\right]=e^{\varphi(\gamma, \cdot)} \delta_{\alpha_{1}}^{\gamma} t_{\alpha_{2} \cdots \alpha_{l}}-t_{\alpha_{1} \cdots \alpha_{l-1}} \delta_{\alpha_{l}}^{\gamma} e^{-\varphi(\cdot, \gamma)}
$$

Proof. (First part.) We shall prove that (2.14) is necessary the "only if" part. Then we shall study the integrability of (2.14). Later, in $\S 6$, we shall complete the proof of Theorem 2. Insert (2.5) into (2.6) and use (2.10). The contribution to $Y B$ in grade $(l, n)$ is

$$
R_{12}^{0} R_{13}^{0} R_{23}^{0} e_{-\alpha_{1}} \cdots e_{-\alpha_{l}} \otimes P_{\alpha_{1} \cdots \alpha_{l}}^{\gamma_{1} \cdots \gamma_{n}} \otimes e_{\gamma_{1}} \cdots e_{\gamma_{n}},
$$

in which $P$ is the sum over $m$, from 0 to $\min (l, n)$, of the following elements of $\mathscr{A}$,

$$
\begin{aligned}
& t_{\alpha_{l}-m+1}^{\gamma_{1} \cdots \gamma_{m}} \cdots \alpha_{l} t_{\alpha_{1} \cdots \alpha_{l-m}} e^{-\varphi(\cdot, \sigma)} t^{\gamma_{m}+1 \cdots \gamma_{n}} \\
& \quad-t_{\alpha_{1} \cdots \alpha_{m}}^{\gamma_{n-m+1} \cdots \gamma_{n}{ }^{\prime} t_{1} \cdots \gamma_{n-m}} e^{\varphi(\tau, \cdot)} t_{\alpha_{m+1} \cdots \alpha_{l}},
\end{aligned}
$$

where $\sigma=\gamma_{1}+\cdots+\gamma_{m}$ and $\tau=\alpha_{1}+\cdots+\alpha_{m}$. The Yang-Baxter relation is satisfied in grade $(l, n)$ if and only if this expression, summed over $m$, vanishes for every index set $\alpha_{1}, \cdots, \alpha_{l}$ and $\gamma_{1}, \cdots, \gamma_{n}$. This is so because $\mathscr{A}^{+}$and $\mathscr{A}^{-}$ are freely generated. We have used the definition (2.13) and

$$
t^{\gamma_{1} \cdots \gamma_{n}}:=t_{\gamma_{n} \cdots \gamma_{n}^{\prime}}^{\gamma_{1} \cdots \gamma_{n}} e-\gamma_{1}^{\prime} \cdots e-\gamma_{n}^{\prime} .
$$

The lowest grades in which $t_{l}=\left(t_{\alpha_{1} \cdots \alpha_{l}}\right)$ appears are $(l, 0)$ and $(0, l)$. In these cases $m=0$ and (2.15) vanishes identically. At grade $(l, 1)$ one finds (summing $m=0,1)$, the linear recursion relation

$$
\left[t_{l}, e-\gamma\right]=e^{\varphi(\gamma, \cdot)} \delta_{\alpha_{1}}^{\gamma} t_{l-1}-t_{l-1} \delta_{\alpha_{l}}^{\gamma} e^{-\varphi(\cdot, \gamma)},
$$

the full expression for which is Eq. (2.14). This equation is therefore necessary. That it is also sufficient will be proved in $\S 6$.

\section{§3. Differential Algebras}

Let $B$ be the unital $C$-algebra freely generated by $\left\{\xi_{i}\right\} i \in N$, countable. Suppose given a map

$$
q: N \times N \rightarrow C, \quad(i, j) \rightarrow q^{i j} \neq 0 .
$$

Introduce the natural grading on $B, B=\oplus B_{n}$, and a set of differential operators

$$
\partial_{i}: B_{n} \rightarrow B_{n-1}, \quad i \in N \text {, }
$$


defined by

$$
\partial_{i} \xi_{j}=\delta_{i}^{i}+q^{i j} \xi_{j} \partial_{i}
$$

We study the problem of integrating sets of equations of the type:

$$
\partial_{i} X=Y_{i}, \quad X \in B, \quad Y_{i} \in B, \quad i \in N .
$$

The collection $\left\{Y_{i}\right\} \quad i \in N$ can be interpreted as the components of a $B$-valued one-form $Y$, on the space $\left\{c^{i} \partial_{i}, c^{i} \in C, i \in N\right\}$. A constant in $B_{n}$ is an element $X \in B_{n}$, such that $\partial_{i} X=0, \forall i \in N$.

Proposition 3.1. (a) The following statements are equivalent: (i) Eq. (3.4) is (uniquely) integrable for every one-form $Y$ with components in $B_{n-1}$. (ii) There are no constants in $B_{n}$. (b) When the parameters $q^{i j}$ are in general position, then there are no constants in $B_{n}, n \geq 1$.

Proof. Let

$$
X=X^{i_{1} \cdots i_{n}} \xi_{i_{1}} \cdots \xi_{n} \in B_{n},
$$

then $\partial_{i} X=0$ means that, for each index set,

$$
\begin{gathered}
X^{i_{1} \cdots i_{n}}+q^{i_{1} i_{2}} X^{i_{2} i_{1} i_{3} \cdots}+q^{i_{1} i_{2}} q^{i_{1} i_{3}} X^{i_{2} i_{3} i_{1} i_{4} \cdots} \\
+\cdots+q^{i_{1} i_{2}} \cdots q^{i_{1} i_{n}} X^{i_{2} \cdots i_{n} i_{1}}=0
\end{gathered}
$$

Now fix the unordered index set $\left\{i_{1}, \cdots, i_{n}\right\}$. If the values are distinct then we have a set of $n$ ! equations for $n$ ! coefficients; in general the number of equations is always equal to the number of unknowns. Solutions exist if and only if the determinant of the matrix of coefficients vanishes. This determinant is an algebraic function of the $q^{i j}$, and not identically zero (since it is equal to 1 when all the $q$ 's vanish), therefore solutions of (3.5), other than $\boldsymbol{X}=0$, exist only on an algebraic subvariety of parameter space.

The calculation of all these determinants will be reported elsewhere. For $n=2$ the result is

$$
D^{12}=1-q^{12} q^{21}, \quad D^{11}=1+q^{11} .
$$

For $n=3$, 


$$
\begin{aligned}
& D^{123}=\left(1-\sigma^{12}\right)\left(1-\sigma^{13}\right)\left(1-\sigma^{23}\right)\left(1-\sigma^{12} \sigma^{13} \sigma^{23}\right) \\
& D^{112}=\left(1+q^{11}\right)\left(1-\sigma^{12}\right)\left(1-q^{11} \sigma^{12}\right) \\
& D^{111}=1+q^{11}+\left(q^{11}\right)^{2}, \quad \sigma^{12}:=q^{12} q^{21}
\end{aligned}
$$

It is natural to pass from $B$ to the quotient by the ideal generated by the constants. In $B_{2}$ the constants are

$$
\begin{array}{ll}
\xi_{1} \xi_{2}-q^{21} \xi_{2} \xi_{1}, & \text { when } \sigma^{12}=1, \\
\xi_{1} \xi_{1}, & \text { when } q^{11}=-1 .
\end{array}
$$

If $q^{i i}=-1, i \in N$ and $\sigma^{i j}=1, i \neq j$, then the quotient is a $q$-Grassmann algebra or quantum antiplane. The constants in $B_{3}$ are

$$
\begin{aligned}
& \xi_{1} \xi_{1} \xi_{1} \quad, 1+q^{11}+\left(q^{11}\right)^{2}=0, \\
& \xi_{1} \xi_{1} \xi_{2}-\left(q^{21}\right)^{2} \xi_{2} \xi_{1} \xi_{1} \quad, 1+q^{11}=0 \text {, } \\
& q^{12} \xi_{1} \xi_{1} \xi_{2}-\left(1+\sigma^{12}\right) \xi_{1} \xi_{2} \xi_{1}+q^{21} \xi_{2} \xi_{1} \xi_{1}, \quad q^{11} \sigma^{12}=1 \text {; }
\end{aligned}
$$

if $\sigma^{12}=1$, there are two constants

$$
\begin{aligned}
& q^{11} \xi_{1} \xi_{1} \xi_{2}-\left(1+q^{11}\right) \xi_{1} \xi_{2} \xi_{1}+\left(q^{21}\right)^{2} \xi_{2} \xi_{1} \xi_{1}, \\
& {\left[\left[\xi_{1}, \xi_{2}\right]_{q^{21}}, \xi_{3}\right]_{q^{31} q^{32}}, \quad[a, b]_{q}:=a b-q b a,}
\end{aligned}
$$

and finally if $\sigma^{12} \sigma^{13} \sigma^{23}=1$ there is one,

$$
\left(\frac{1}{q^{31}}-q^{13}\right)\left(\xi_{1} \xi_{2} \xi_{3}+q^{31} q^{32} q^{21} \xi_{3} \xi_{2} \xi_{1}\right)+\text { cyclic }
$$

Annulment of (3.8), (3.12) and (3.13) are $q$-deformed Serre relations [D1]. The last item, Eq. (3.15), may be something new; it should be interesting to study the quotient of the algebra $B$ with 3 generators by the ideal generated by this constant.

A constant that involves only one variable, $\xi_{1}$ say, exists if and only if $q^{11} \neq 1$ is a root of unity,

$$
\xi_{1}^{n} \text { constant iff }\left(q^{11}\right)^{n}=1, n=2,3, \cdots, q \neq 1 .
$$

It is easy to determine all constants of the $q$-Serre type; that is, all those that involve two generators and one of them linearly, 


$$
C:=\sum_{m=0}^{k} Q_{m}^{k}\left(\xi_{1}\right)^{m} \xi_{2}\left(\xi_{1}\right)^{k-m}=0
$$

With $q=q^{11}$,

$$
\partial_{1}\left(\xi_{1}\right)^{m}=(m)_{q}\left(\xi_{1}\right)^{m-1}, \quad(m)_{q}:=1+q+\cdots+q^{m-1} .
$$

Setting $\partial_{1} C=0$ gives, for $q^{n} \neq 1, n \in Z$,

$$
Q_{m}^{k}=\left(-q^{12}\right)^{m} q^{m(m-1) / 2}\left(\begin{array}{l}
k \\
m
\end{array}\right)_{q}
$$

while $\partial_{2} C=0$ is equivalent to

$$
\prod_{m=0}^{k-1}\left(1-q^{m} \sigma^{12}\right)=0
$$

When $k=2$, compare $D^{112}$ in Eq. (3.7). If $k$ is the smallest integer such that a relation like (3.16) holds, then

$$
1-q^{k-1} \sigma^{12}=0
$$

Here are some partial results for $B_{4}$ and $B_{5} . D_{1234}$ is the product of 12 factors of the form $1-\sigma_{i j}, 4$ factors of the form $1-\sigma_{i j} \sigma_{k l} \sigma_{m n}, 2$ identical factors of the form $1-\sigma_{12} \cdots \sigma_{34}$; each group accounts for 24 orders in the $q$ 's. $D_{12345}$ is the product of 60 factors of the first type, 20 factors of the second type, 10 factors of the third type and 6 identical factors of the form 1 -(product of all ten $\sigma_{i j}, i \neq j$ ); each group accounts for 120 orders in the $q$ 's. Finally the following is true.

Proposition 3.2. If all (finite) products of the form $\Pi_{i, j}\left(q_{i j}\right)^{n_{i j}}$, where $n_{i j}$ are nonnegative integers, differ from unity, then the determinant of the matrix of coefficients in (3.5) is different from zero.

\section{§4. Differential Complexes}

In the generic case, when there are no constants in $B_{n}$, the equation $\partial_{i} X=Y_{i}, \quad Y_{i} \in B_{n-1}, \quad i \in N$, is always solvable, for any one-form $Y$. All one-forms are exact, to be closed has no meaning and the differential complex is highly trivial.

The existence of a constant $C \in B_{n}$ implies that there are one-forms valued in $B_{n-1}$ that are not exact. To each 1-dimensional space of constants in $B_{n}$ there is a one-dimensional space of non-exact one-forms, valued in $B_{n-1}$, 
defined modulo exact one-forms and obtainable as a limit of $\partial_{i} X$ as $X \rightarrow C$, after factoring out a constant. Thus,

$$
X=\xi_{1} \xi_{2}-q^{21} \xi_{2} \xi_{1}
$$

becomes a constant as $\sigma^{12} \rightarrow 1$, and a representative of the associated class of non-exact one-forms is given by

$$
Y_{i}=\lim \left(1-\sigma^{12}\right)^{-1} \partial_{i} X= \begin{cases}\xi_{2}, & i=1 \\ 0, & i \neq 1\end{cases}
$$

Definition 4.1. An elementary constant is a linear combination of re-orderings (permutations of the order of the factors) of a fixed monomial.

A constant $C \in B_{n}$ also implies a concept of closed one-forms.

Proposition 4. If $C \in B_{n}$,

$$
C=C^{i_{1} \cdots i_{n}} \xi_{i_{1}} \cdots \xi_{i_{n}},
$$

is a constant, then the differential operator

$$
\Phi(C):=C^{i_{1} \cdots i_{n}} \partial_{i_{1}} \cdots \partial_{i_{n}}
$$

vanishes on $B$.

Proof. A constant in $B_{n}$ is a sum of elementary constants; it is enough to prove the proposition for the case that $C$ is an elementary constant. This implies that there are non-zero $f_{i} \in C, i \in N$, such that the following operator identity

$$
\partial_{i} C-f_{i} C \partial_{i}=0, \quad i \in N \text {, }
$$

holds on $B$. Let $B^{*}$ be the unital, associative algebra freely generated by $\left\{\partial_{i}\right\}$ $i \in N$, and let $\Phi: B \rightarrow B^{*}$ be the unique isomorphism of algebras such that $\Phi\left(\xi_{i}\right)=\partial_{i}$. Let $B B^{*}(q)$ be the unital, associative algebra generated by $\left\{\xi_{i}, \partial_{i}\right\}$ $i \in N$, with relations (3.3); then $\Phi$ extends to a unique isomorphism

$$
\Phi^{\prime}: B B^{*}(q) \rightarrow B B^{*}(\hat{q}), \quad \hat{q}^{i j}=1 / q^{j i}
$$

In particular, $\Phi^{\prime}\left(\xi_{i}\right)=\partial_{i}$ and $\Phi^{\prime}\left(\partial_{i}\right)=-\left(q^{i i}\right)^{-1} \xi_{i}, i \in N$. Now Eq. (4.5) means 
that $\partial_{i} \circ C=C f_{i} \circ \partial_{i}$, where $a \circ b$ denotes the product in $B B^{*}(q)$. Applying $\Phi^{\prime}$ one gets

$$
\Phi(C) \circ \xi_{i}=\left(f_{i}\right)^{-1} \xi_{i} \circ \Phi(C)
$$

implying that $\Phi(C) X=0, X \in B$.

Definition 4.2. Let $C$ be an elementary constant in $B_{n}, n \geq 2$. A $B_{1}^{*}$ one-form $Y$, valued in $B$, will be said to be $C$-closed if

$$
d_{C} Y:=C^{i_{1} \cdots i_{n}} \partial_{i_{1}} \cdots \partial_{i_{n-1}} Y_{i_{n}}=0 .
$$

Examples. In $B_{2}$ the constants are of type $C=\xi_{1} \xi_{1}$ or $C^{\prime}=\xi_{1} \xi_{2}-q^{21} \xi_{2} \xi_{1}$. Now $Y$ is $C$-closed if $d Y:=\partial_{1} Y_{1}=0$ and $C^{\prime}$-closed if $d^{\prime} Y:=\partial_{1} Y_{2}-q^{21} \partial_{2} Y_{1}=0$. The first case is characteristic of Grassmann algebras and the other of quantum planes. Let $\mathscr{C}$ be the collection

$$
\left\{C_{i j}=\xi_{i} \xi_{j}-q^{j i} \xi_{j} \xi_{i}, \quad i, j \in N, \quad i \neq j\right\}
$$

and suppose all of them constant. (In other words, $q^{i j} q^{j i}=1, i \neq j$.) Then we say that a one-form $Y$ is $\mathscr{C}$-closed if $Y$ is $C_{i j}$-closed for all $i \neq j$ :

$$
\partial_{i} Y_{j}-q^{j i} \partial_{j} Y_{i}=0, \quad i, j \in N, \quad i \neq j
$$

In this case the closure of a $B_{1}^{*}$ one-form is expressed in terms of the $B_{1}^{*}$ two-form

$$
Z=d Y, \quad Z_{i j}=\partial_{i} Y_{j}-q^{j i} \partial_{j} Y_{i},
$$

and this naturally leads to familiar $q$-deformed de Rham complexes, with trivial cohomology. (Non-trivial cohomology depends on completion of the algebra.)

It would be interesting to develop the analogue of this construction, the $q$-deformed de Rham complex, in the more general case when $\mathscr{C}$ is an arbitrary collection of constants. As a highly non-trivial example consider the following. Replace the constants $C_{i j}$ in (4.7), they are Serre relations of order one, with Serre relations of order two:

$$
C_{i j}=q^{i j} \xi_{i} \xi_{i} \xi_{j}-\left(1+\sigma^{i j}\right) \xi_{i} \xi_{j} \xi_{i}+q^{j i} \xi_{j} \xi_{i} \xi_{i}
$$

This implies that $q^{i i}=q^{j j}=1 / \sigma^{i j}, i \neq j$. Then Definition 4.2 says that $Y=\left(Y_{1}, Y_{2}, \cdots\right)$ is a closed one-form if

$$
(d Y)_{i j}:=q^{i j} \partial_{i} \partial_{i} Y_{j}-\left(1+\sigma^{i j}\right) \partial_{i} \partial_{j} Y_{i}+q^{j i} \partial_{j} \partial_{i} Y_{i}=0
$$


Every exact one-form is closed by Proposition 4; the converse statement is less obvious. And then there is this problem: what is the integrability condition for the following set of equations

$$
(d Y)_{i j}=Z_{i j}, i, j \in N
$$

In other words, what two-forms are "closed"?

\section{§5. Integrability of Eq. (2.14)}

It was seen, in Section 2, that a necessary condition for the standard R-matrix (2.5) to satisfy the Yang-Baxter relation (2.6) is that the coefficients $t_{(\alpha)}^{\left(\alpha^{\prime}\right)}$ satisfy the recursion relation (2.14),

$$
\begin{aligned}
& {\left[t_{\alpha_{1} \cdots \alpha_{l}}, e-\gamma\right]=e^{\varphi(\gamma, \cdot)} \delta_{\alpha_{1}}^{\gamma} t_{\alpha_{2} \cdots \alpha_{l}}-t_{\alpha_{1} \cdots \alpha_{l}-1} \delta_{\alpha_{l}}^{\gamma} e^{-\varphi(\cdot, \gamma)},} \\
& t_{\alpha_{1} \cdots \alpha_{l}}:=t_{\alpha_{1} \cdots \alpha_{l}}^{\alpha_{1}^{\prime} \cdots \alpha_{l}} e_{\alpha_{1}^{\prime}} \cdots e_{\alpha_{l}} .
\end{aligned}
$$

Define* two differential operators, $\vec{\partial}_{-\gamma}$ and $\stackrel{\leftarrow}{\partial}_{-\gamma}$, on $\mathscr{A}^{+}$, by

$$
[X, e-\gamma]=e^{\varphi(\gamma, \cdot)} \vec{\partial}_{-\gamma} X-X \overleftarrow{\partial}_{-\gamma} e^{-\varphi(\cdot, \gamma)}
$$

$X \in \mathscr{A}^{+}$; note that $\overleftarrow{\partial}_{-\gamma}$ operates from the right. Similarly,

$$
\left[e_{\alpha}, Y\right]=\overleftarrow{Y \partial_{\alpha}} e^{\varphi(\alpha \cdot)}-e^{-\varphi(\cdot \alpha)} \vec{\partial}_{\alpha} Y
$$

defines two differential operators on $\mathscr{A}^{-}$. These definitions are equivalent to the rules

$$
\begin{array}{ll}
\vec{\partial}_{-\gamma} e_{\alpha}=\delta_{\alpha}^{\gamma}+e^{-\varphi(\gamma, \alpha)} e_{\alpha} \vec{\partial}_{-\gamma}, & e_{\alpha} \overleftarrow{\partial}_{-\gamma}=\delta_{\alpha}^{\gamma}+e^{-\varphi(\alpha, \gamma)} \overleftarrow{\partial}_{-\gamma} e_{\alpha}, \\
e_{-\alpha} \overleftarrow{\partial}_{\gamma}=\delta_{\alpha}^{\gamma}+e^{-\varphi(\gamma, \alpha)} \overleftarrow{\zeta}_{\gamma} e_{-\alpha}, & \vec{\partial}_{\gamma} e_{-\alpha}=\delta_{\alpha}^{\gamma}+e^{-\varphi(\alpha . \gamma)} e_{-\alpha} \vec{\partial}_{\gamma}
\end{array}
$$

Eq. (5.1) is equivalent to

$$
\vec{\partial}_{-\gamma} t_{\alpha_{1} \cdots \alpha_{l}}=\delta_{\alpha_{1}}^{\gamma} t_{\alpha_{2} \cdots \alpha_{l}}, \quad t_{\alpha_{1} \cdots \alpha_{l}} \stackrel{\leftarrow}{\partial}_{-\gamma}=t_{\alpha_{1} \cdots \alpha_{l-1}} \delta_{\alpha_{l}}^{\gamma} .
$$

Proposition 5.1. Suppose that the parameters $\varphi(\alpha, \beta)$ are in general position, so that there are no constants in $\mathscr{A}^{+}\left(\mathscr{A}^{-}\right)$with respect to the differential operators $\vec{\partial}_{-\gamma}$ or $\overleftarrow{\partial}_{-\gamma}\left(\vec{\partial}_{\gamma}\right.$ or $\left.\overleftarrow{\partial}_{\gamma}\right)$. Then either one of the two equations in (5.5) determines $t_{\alpha_{1} \cdots \alpha_{l}}$ recursively and uniquely (the same in each case) from $t_{\alpha}=e_{\alpha}$.

\footnotetext{
* This is where we need the last condition in Definition 2.1.
} 
Proof. From (5.4) we deduce that

$$
\left.\vec{\partial}_{-\gamma} X\right) \overleftarrow{\partial}_{-\gamma^{\prime}}=\vec{\partial}_{-\gamma}\left(X \overleftarrow{\partial}_{-\gamma^{\prime}}\right)
$$

By Proposition 3, the first of (5.5) determines $t_{l}=t_{\alpha_{1} \cdots \alpha_{l}}$ uniquely from $t_{\alpha}=e_{\alpha}$. The other recursion relation also has a unique solution, $t_{l}^{\prime}$ say. We must show that $t_{l}=t_{l}^{\prime}, l>1$. Since parentheses are superfluous,

$$
\begin{aligned}
& \vec{\partial}_{-\gamma} t_{l} \overleftarrow{\partial}_{-\gamma^{\prime}}=\delta_{\alpha_{1}}^{\gamma} t_{l-1} \overleftarrow{\partial}_{-\gamma^{\prime}}, \\
& \vec{\partial}_{-\gamma} t_{l}^{t_{l} \overleftarrow{\partial}_{-\gamma^{\prime}}}=\vec{\partial}_{-\gamma} t_{l-1}^{\prime} \delta_{\alpha_{l}}^{\gamma^{\prime}} .
\end{aligned}
$$

Suppose $t_{k}^{\prime}=t_{k}$ for $k=1, \cdots, l-1$; then the right-hand sides are both equal to $\delta_{\alpha_{1}}^{\gamma} t_{l-2} \delta_{\alpha_{l}}^{\gamma^{\prime}}$. Then the left-hand sides are also equal and, since there are no constants, $t_{l}=t_{l}^{\prime}$. Since $t_{1}=t_{1}^{\prime}\left(t_{\alpha}=t_{\alpha}^{\prime}=e_{\alpha}\right)$, the proposition follows by induction.

We also encounter the relation

$$
\begin{aligned}
& {\left[e_{\alpha}, t^{\gamma_{1} \cdots \gamma_{n}}\right]=t^{\gamma_{1} \cdots \gamma_{n-1}} \delta_{\alpha}^{\gamma_{n}} e^{\varphi(\alpha, \cdot)}-e^{-\varphi(\cdot, \alpha)} \delta_{\alpha}^{\gamma_{1}} t^{\gamma_{2} \cdots \gamma_{n}},} \\
& t^{\gamma_{1} \cdots \gamma_{n}}:=t_{\gamma_{1}^{\prime} \cdots \gamma_{n}^{\prime}}^{\gamma_{1} \cdots \gamma_{n}} e_{-\gamma_{1}^{\prime}} \cdots e_{-\gamma_{n}^{\prime}} .
\end{aligned}
$$

Just as (5.1) is equivalent to (5.5), this relation is the same as

$$
t^{\gamma_{1} \cdots \gamma_{n}} \stackrel{\leftarrow}{\partial_{\alpha}}=t^{\gamma_{1} \cdots \gamma_{n-1}} \delta_{\alpha}^{\gamma_{n}}, \quad \overrightarrow{\partial_{\alpha}} t^{\gamma_{1} \cdots \gamma_{n}}=\delta_{\alpha}^{\gamma_{1}} t^{\gamma_{2} \cdots \gamma_{n}}
$$

An adaptation of the proof of Proposition 5.1 shows that either one of these two relations determines the same set $t_{\alpha_{1}, \cdots, \alpha_{n}}$. Finally, we verify that this unique solution of (5.8) coincides with the solution of (5.1).

Proposition 5.2. Fix an unordered set $\left\{\alpha_{1} \cdots, \alpha_{n}\right\}$ and let $S$ be the matrix (with entries in $C$ ) defined by the natural pairing between the algebras generated by the $e_{\alpha}$ 's and the $\vec{\partial}_{-\beta}$ 's, respectively,

$$
S_{(\alpha)}^{(\beta)}=\vec{\partial}_{-\beta_{n}} \cdots \vec{\partial}_{-\beta_{1}} e_{\alpha_{1}} \cdots e_{\alpha_{n}},
$$

where $(\alpha),(\beta)$ run over the ordered sets that coincide as unordered set with $\left\{\alpha_{1}, \cdots, \alpha_{n}\right\}$. Similarly,

$$
S_{(\alpha)}^{\prime(\beta)}=\vec{\partial}_{\alpha_{n}} \cdots \vec{\partial}_{\alpha_{1}} e_{-\beta_{1}} \cdots e_{-\beta_{n}}
$$

Then (a) $S=S^{\prime}$ and (b) the matrix $t=t_{(\alpha)}^{(\beta)}$ is given by

$$
S t=t S^{\prime}=1 \text {. }
$$


Proof. (a) By inspection: Moving the operator $\vec{\partial}_{-\beta_{1}}$ to the right till it encounters and annihilates $e_{\beta_{1}}$ produces a factor $\Pi_{\alpha} \exp \left(-\varphi\left(\beta_{1}, \alpha\right)\right)$, where the product runs over those $e_{\alpha}$ 's that stand to the left of $e_{\beta_{1}}$. The same factor is produced by moving $e_{-\beta_{1}}$ to the left in the expression for $S^{\prime}$. (b) Iteration of the first of Eq.s (5.5) yields $S t=1$ and iteration of the second of (5.8) gives $t S^{\prime}=1$. This shows, in particular, that (5.5) and (5.8) are solvable under the same conditions; namely, if and only if the matrix $S$ is invertible.

\section{§6. Completion of the Proof of Theorem 2}

Suppose that the relations (2.14) are satisfied for $l \geq 1$. Now fix $l, n$, $\alpha_{1}, \cdots, \alpha_{l}$ and $\gamma^{1}, \cdots, \gamma^{n}$; we must prove that the expression (2.15), summed over $m$ from 0 to $\min (l, n)$, vanishes.

We begin by calculating the sum over $m=0,1$ (step 1); then we postulate a formula for the partial sum over $m=0, \cdots, k$ (step $k$ ). We prove the formula by induction in $k$, and finally show that the sum vanishes when $k=\min (l, n)$.

The term $m=0$ in $(2.15)$ is

$$
\left[t_{l}, t^{n}\right]=t_{\gamma_{1}^{\prime} \cdots \gamma_{n}^{\prime}}^{\gamma_{1} \cdots \gamma_{n}} \sum_{i=1}^{n} e_{-\gamma_{1}^{\prime}} \cdots e_{-\gamma_{i-1}^{\prime}}\left[t_{l}, e_{-\gamma_{i}^{\prime}}\right] e_{-\gamma_{i+1}^{\prime}} \cdots e_{-\gamma_{n}^{\prime}}
$$

As in the preceding section we often write $t_{l}, t^{n}$ for $t_{\alpha_{1} \cdots \alpha_{l}}, t^{\gamma_{1} \cdots \gamma_{n}}$. We shall gradually make the formulas more schematic so as to bring out their structure. By (2.14)

$$
=t_{\left(\gamma^{\prime}\right)}^{(\gamma)} \sum_{i=1}^{n} e_{-\gamma_{1}^{\prime}} \cdots\left(e^{\varphi\left(\gamma_{1}^{\prime}, \cdot\right)} \delta_{\alpha_{1}}^{\gamma_{1}^{\prime}} t_{l-1}-t_{l-1} \delta_{\alpha_{l}}^{\gamma_{i}^{\prime}} e^{-\varphi\left(\cdot, \gamma_{i}^{\prime}\right)}\right) \cdots e_{-\gamma_{n}^{\prime}}
$$

The term $m=1$ is

$$
\begin{aligned}
& t_{\alpha_{l}}^{\gamma_{1}} t_{\alpha_{1} \cdots \alpha_{l}-1} e^{-\varphi\left(\cdot, \gamma_{1}\right)} t^{\gamma_{2} \cdots \gamma_{n}}-t_{\alpha_{l}}^{\gamma_{n}} t^{\gamma_{1} \cdots \gamma_{n-1}} e^{\varphi\left(\alpha_{1}, \cdot\right)} t_{\alpha_{2} \cdots \alpha_{l}} \\
= & t_{l-1} e^{-\varphi\left(\cdot, \alpha_{l}\right)} \vec{\partial}_{\alpha_{l}} t^{\gamma_{1} \cdots \gamma_{n}}-t^{\gamma_{1} \cdots \gamma_{n}} \overleftarrow{\partial}_{\alpha_{1}} e^{\varphi\left(\alpha_{1}, \cdot\right)} t_{l-1} \\
= & t_{l-1} t_{\left(\gamma^{\prime}\right)}^{(\gamma)} \sum e_{-\gamma_{1}^{\prime}} \cdots \delta_{\alpha_{l}}^{\gamma_{i}^{\prime}} e^{-\varphi\left(\cdot, \gamma_{i}^{\prime}\right) \cdots} e_{-\gamma_{n}^{\prime}}-t_{\left(\gamma^{\prime}\right)}^{(\gamma)} \sum e_{-\gamma_{1}^{\prime}} \cdots \delta_{\alpha_{1}}^{\gamma_{i}^{\prime}} e^{\varphi\left(\gamma_{i}^{\prime} \cdot \cdot\right)} \cdots e_{-\gamma_{n}^{\prime}} t_{l-1}
\end{aligned}
$$

This agrees with (6.2) except for the position of $t_{l-1}$, and the sign. Thus, adding the contributions $m=0,1$ we obtain

$$
\begin{aligned}
t_{\left(\gamma^{\prime}\right)}^{(\gamma)} \sum_{i<j}\left\{e_{-\gamma_{1}^{\prime}} \cdots \delta_{\alpha_{1}^{\prime}}^{\gamma_{i}^{\prime}} e^{\varphi\left(\gamma_{i}^{\prime}, \cdot\right)} e_{-\gamma_{i+1}^{\prime}} \cdots\left[t_{l-1}, e_{-\gamma_{j}^{\prime}}\right] \cdots e_{-\gamma_{n}^{\prime}}\right. \\
\quad+e_{-\gamma_{1}^{\prime}} \cdots\left[t_{l-1}, e_{-\gamma_{i}^{\prime}}\right] e_{-\gamma_{i+1}^{\prime}} \cdots \delta_{\alpha_{l}}^{\gamma_{j}^{\prime}} e^{\left.-\varphi\left(\cdot, \gamma_{j}^{\prime}\right) \cdots e_{-\gamma_{n}^{\prime}}\right\}}
\end{aligned}
$$


This completes step 1; all terms involving $t_{l}$ have disappeared and $t_{l-1}$ appears only in commutators that allow us to use (2.14) again.

We claim that after carrying out step $k$, which includes summing over $m=0, \cdots, k$, one obtains the following expression

$$
\sum_{s=0}^{k} \cdots\left(\delta e^{\varphi}\right)^{k-s} \cdots\left[t_{l-k}, e-\gamma\right] \cdots\left(\delta e^{-\varphi}\right)^{s} \cdots, k<\min (l, n)
$$

and zero, $k=\min (l, n)$. Here the dots stand for products of the $e_{-\gamma_{i}^{i}}$, interrupted $k-s$ times by a factor of the type $\delta_{\alpha_{1}}^{\gamma_{i}^{i}} e^{\varphi\left(\gamma_{i}^{\prime}, \cdot\right)}$, once by [,] and $s$ times by a factor like $\delta_{\alpha_{l}}^{\gamma_{i}} e^{-\varphi\left(\cdot, \gamma_{i}^{i}\right)}$, as in (6.3).

To verify this claim we carry out the next step. We first evaluate the commutators and examine the cancellations that take place between successive terms in the sum (6.4):

$$
\begin{aligned}
& \cdots {\left[t_{l-k}, e-\gamma_{i}\right] \cdots\left(\delta e^{-\varphi}\right) \cdots+\cdots\left(\delta e^{\varphi}\right) \cdots\left[t_{l-k}, e_{-\gamma_{i}}\right] \cdots } \\
&=\cdots\left(\delta_{\alpha}^{\gamma_{i}^{i}} e^{\varphi(\alpha, \cdot)} t_{l-k-1}-t_{l-k-1} \delta_{\alpha}^{\gamma^{\prime}} e^{-\varphi(\cdot, \alpha)}\right) \cdots\left(\delta e^{-\varphi}\right) \cdots \\
& \quad+\left(\delta e^{\varphi}\right) \cdots\left(\delta_{\alpha}^{\gamma_{j}^{\prime}} e^{\varphi(\alpha, \cdot)} t_{l-k-1}-t_{l-k-1} \delta_{\alpha}^{\gamma^{\prime} j} e^{-\varphi\left(\cdot, \alpha^{\prime}\right)}\right) \cdots
\end{aligned}
$$

The first term in the first line combines with the second term in the second line to

$$
\cdots\left(\delta e^{\varphi}\right) \cdots\left[t_{l-k-1}, e_{-\gamma}\right] \cdots\left(\delta e^{-\varphi}\right) \cdots
$$

Successive terms in (6.4) all combine in this way, to reproduce the same expression with $k$ replaced by $k+1$, except for the fact that there is no term in the sequence that precedes and collaborates with the first term and no term that succeeds and collaborates with the last term. It remains, therefore, to be proved that the summand $m=k+1$ in (2.15) precisely supplies the two missing terms.

By (5.8),

$$
\begin{aligned}
& \vec{\partial}_{\beta} t^{\gamma_{1} \cdots \gamma_{n}}=\delta \beta^{\gamma_{1}} t^{\gamma_{2} \cdots \gamma_{n}}, \\
& \vec{\partial}_{\beta_{m}} \cdots \vec{\partial}_{\beta_{1}} t^{\gamma_{1} \cdots \gamma_{n}}=\delta \delta_{1}^{\gamma_{1}} \cdots \delta_{\beta_{m}^{m}}^{\gamma_{m}} t^{\gamma_{m}+1 \cdots \gamma_{n}}, \\
& t_{\alpha_{l-m} \cdots \beta_{m} \cdots \alpha_{l}}^{\beta_{1} \cdots \beta_{\beta_{m}}} \cdots \vec{\partial}_{\beta_{1}} t^{\gamma_{1} \cdots \gamma_{n}}=t_{\alpha_{l}-m+1}^{\gamma_{1} \cdots \gamma_{m} \cdots \alpha_{l}} t^{\gamma_{m+1} \cdots \gamma_{n}} .
\end{aligned}
$$

Hence, if $\vec{t}_{\alpha_{1} \ldots \alpha_{l}}$ is the differential operator

$$
\vec{t}_{\alpha_{1} \cdots \alpha_{l}}:=t_{\alpha_{1} \cdots \alpha_{l}}^{\alpha_{1}^{\prime} \cdots \alpha_{\alpha i}} \vec{\partial}_{\alpha_{1}^{\prime}} \cdots \vec{\partial}_{\alpha_{1}^{\prime}},
$$

then 


$$
t_{\alpha_{l-m+1} \cdots \alpha_{l}}^{\gamma_{1} \cdots \gamma_{m}} t^{\gamma_{m+1} \cdots \gamma_{n}}=\vec{t}_{\alpha_{l} \cdots \alpha_{l-m+1}} t^{\gamma_{1} \cdots \gamma_{n}}
$$

and the first of the two terms in (2.15) is

$$
\begin{aligned}
& t_{\alpha_{1} \cdots \alpha_{l}-m} e^{-\varphi(\cdot, \sigma)} \vec{t}_{\alpha_{l} \cdots \alpha_{l-m+1}} t^{\gamma_{1} \cdots \gamma_{n}} \\
& =t_{\alpha_{1} \cdots \alpha_{l}-m} t\left(\gamma_{\gamma^{\prime}}^{(\gamma)}\right) \sum_{i=1}^{n} e_{-\gamma_{1}} \cdots\left[e^{-\varphi(\cdot, \sigma)}{\overrightarrow{t_{\alpha}} \cdots \alpha_{l-m+1}}, e-\gamma_{i}\right] \cdots \\
& =t_{\alpha_{1} \cdots \alpha_{l-m}} t\left(\gamma_{\gamma}^{\gamma}\right) \sum_{l} e_{-\gamma_{1}^{\prime}} \cdots \delta_{\alpha_{l-m+1}}^{\gamma_{i}^{i}} e^{-\varphi(\cdot, \sigma)} \vec{t}_{\alpha_{l} \cdots \alpha_{l-m+2}} \cdots
\end{aligned}
$$

By iteration of these steps one finally ends up, when $m=k+1$, with precisely the missing terms; actually one of the missing terms, we leave it to the reader to carry out the calculation for the other one. This done, the proof of Theorem 2 is complete.

Corollary 6. With the parameters in general position, there exists a unique standard R-matrix on $\mathscr{A}$ that satisfies the Yang-Baxter relation.

\section{§7. Obstructions and Generalized Serre Relations}

We have been concerned with the construction of a standard R-matrix, Definition (2.2), that satisfies the Yang-Baxter relation, Eq.(2.6), on an algebra $\mathscr{A}$, Definition (2.1). The relations of $\mathscr{A}$ involve parameters; when these parameters are in general position then the recursion relation (2.14) has a unique solution that provides the unique standard R-matrix on $\mathscr{A}$ that satisfies the Yang-Baxter relation. At certain hypersurfaces in parameter space we have encountered obstructions, characterized by the vanishing of one or more of the determinants that we have studied in $\S 3$. At these points there appear elements in $\mathscr{A}^{+}$that are constants with respect to differential operators $\vec{\partial}_{-\alpha}$ and $\overleftarrow{\partial}_{-\alpha}$, and elements in $\mathscr{A}^{-}$that are constans with respect to $\vec{\partial}_{\alpha}$ and $\overleftarrow{\partial}_{\alpha}$. Then there is no solution of $(2.14)$ and no standard R-matrix on $\mathscr{A}$ that satisfies Yang-Baxter.

We shall show that all these obstructions can be overcome by the introduction of additional relations in the definition of $\mathscr{A}$ or, what is the same, by replacing $\mathscr{A}$ by a quotient $\mathscr{A} / I$, where $I$ is the ideal generated by the constants. The next three propositions relate the null-spaces of the four differential operators to each other. 
Proposition 7.1. The space of constants with respect to $\vec{\partial}_{-\gamma}$ in $\mathscr{A}_{n}^{+}$has the same dimension as the space of constants with respect to $\overleftarrow{\partial}_{-\gamma}$. If there are no constants in $\mathscr{A}_{l}^{+}$for $l=1, \cdots, n-1$, then the two spaces coincide.

Proof. An easy consequence of Eq. (5.6).

Let $C \in \mathscr{A}_{n}^{+}$be a constant with respect to $\vec{\partial}_{-\gamma}, \gamma \in N$, and assume, provisionally, that there are no constants in $\mathscr{A}_{l}^{+}, 1<l<n$. Without essential loss of generality we take $C$ to be a linear combination

$$
C=C^{\gamma_{1} \cdots \gamma_{n}} e_{\gamma_{1}} \cdots e_{\gamma_{n}}
$$

where the summation runs over the permutations of a fixed set $\left\{\gamma_{1} \cdots \gamma_{n}\right\}$, hence over a finite set. Let $d$ be the operator that takes $X \in \mathscr{A}_{n}^{+}$to the one-form $Y$ valued in $\mathscr{A}_{n-1}^{+}$with components $\vec{\partial}_{-\gamma} X, \gamma \in N$. This operator is represented by a direct sum of finite square matrices, also denoted $d$. The constant $C$ is a null-vector for $d$. The transposed matrix also has a null-vector; it exists by virtue of the fact that $d X$ is $C$-closed: (Definition 4.2 ):

$$
\begin{aligned}
& \left(C^{\gamma_{1} \cdots \gamma_{n}} \vec{\partial}_{-\gamma_{1}} \cdots \vec{\partial}_{-\gamma_{n}}\right) e_{\alpha_{1}} \cdots e_{\alpha_{n}} \\
= & \left(C^{\gamma_{1} \cdots \gamma_{n}} \vec{\partial}_{-\gamma_{1}} \cdots \vec{\partial}_{-\gamma_{n-1}}\right)\left(d_{\alpha_{1} \cdots \alpha_{n}}^{\gamma_{n} \beta_{1} \cdots \beta_{n-1}} e_{\beta_{1}} \cdots e_{\beta_{n-1}}\right) \\
= & d_{\alpha_{1} \cdots \alpha_{n}}^{\gamma_{n} \beta_{1} \cdots \beta_{n-1}}\left(C^{\gamma_{1} \cdots \gamma_{n}} \vec{\partial}_{-\gamma_{1}} \cdots \vec{\partial}_{-\gamma_{n-1}} e_{\beta_{1}} \cdots e_{\beta_{n-1}}\right)=0 .
\end{aligned}
$$

The obstruction to solving Eq. (5.5) is that the right-hand side is not in the null-space of the transpose of $d$; it is not $C$-closed. Indeed, since there are no constants in $\mathscr{A}_{l}^{+}, l<n$,

$$
\left(C^{\gamma_{1} \cdots \gamma_{n}} \vec{\partial}_{-\gamma_{1}} \cdots \vec{\partial}_{-\gamma_{n-1}}\right) \delta_{\gamma_{n}}^{a_{1}} t_{\alpha_{2} \cdots \alpha_{n}}=C^{\alpha_{n} \cdots \alpha_{1}} \neq 0 .
$$

Recall that the R-matrix is expressed in terms of $e_{-\alpha_{1}} \cdots e_{-\alpha_{n}} t_{\alpha_{1} \cdots \alpha_{n}}$. The obstruction to Yang-Baxter is thus

$$
e_{-\alpha_{1}} \cdots e-\alpha_{n} C^{\alpha_{n} \cdots \alpha_{1}}=: C^{\prime} \in \mathscr{A}_{n}^{-} .
$$

Proposition 7.2. The element $C^{\prime} \in \mathscr{A}_{n}^{-}$is a constant.

Proof. One verifies directly that $\vec{\partial}_{-\gamma} C=0, \gamma \in N$, is equivalent to $C^{\prime} \overleftarrow{\partial}_{\gamma}=0$ $\gamma \in N$.

Thus, if the first obstruction to the Yang-Baxter relation is encountered 
at the evaluation of $t_{\alpha_{1} \cdots \alpha_{n}}$, then this obstruction can be avoided by replacing $\mathscr{A}$ by the quotient $\mathscr{A} / I_{n}$, where $I_{n}$ is the ideal generated by the constants in $\mathscr{A}_{n}^{ \pm}$. Once this has been done, we study the obstructions at the next level. Since the constants at level $n$ have been removed there are none in $\mathscr{A}_{t}^{ \pm}, l \leq n$, and substantially the same analysis applies to constants in $\mathscr{A}_{n+1}^{ \pm}$. To formulate the final result we need:

Proposition 7.3. The ideal $I^{+} \subset \mathscr{A}^{+}$generated by the constants of $\vec{\partial}_{-\gamma}$, coincides with that generated by the constants of $\overleftarrow{\partial}_{-\gamma}$. The same statement holds true in $\mathscr{A}^{-}$, mutatis mutandi.

When the parameters are in general position there are no constants, and Theorem 2 with Proposition 5 assures us that there is a unique standard R-matrix in $\mathscr{A} \otimes \mathscr{A}$ that satisfies the Yang-Baxter relation (2.6). We are now in a position to allow for the appearance of constants.

Remark. There are no constants in $\mathscr{A}_{1}^{ \pm}$; the generators $H_{a}, e_{ \pm \alpha}$ of $\mathscr{A}$ are also generators of $\mathscr{A}^{\prime}=\mathscr{A} / I$.

Theorem 7. Let $I \subset \mathscr{A}$ be the ideal generated by the constants in $\mathscr{A}^{+}$ and the constants in $\mathscr{A}^{-}$, and let $\mathscr{A}^{\prime}$ be the quotient $\mathscr{A} / I$. Interpret the standard, universal $R$-matrix (2.5) as an element of $\mathscr{A}^{\prime} \otimes \mathscr{A}^{\prime}$. The Yang-Baxter relation for the standard $R$-matrix on $\mathscr{A}^{\prime}$ is equivalent to the recursion relation

$$
\begin{aligned}
{\left[t_{l}, 1 \otimes e_{-\gamma}\right] } & =\left(e_{-\gamma} \otimes e^{\varphi(\gamma, \cdot)}\right) t_{l-1}-t_{l-1}\left(e_{-\gamma} \otimes e^{-\varphi(\cdot, \gamma)}\right), \\
t_{l} & :=t_{(\alpha)}^{\left(\alpha^{\prime}\right)} e_{-\alpha_{1}} \cdots e_{-\alpha_{l}} \otimes e_{\alpha_{1}^{\prime}} \cdots e_{\alpha_{l}^{\prime}},
\end{aligned}
$$

and to either one of the following

$$
\begin{gathered}
{\left[e_{\gamma}, t_{l} \otimes 1\right]=t_{l-1}\left(e^{\varphi(\gamma \cdot)} \otimes e_{\gamma}\right)-\left(e^{-\varphi(\cdot, \gamma)} \otimes e_{\gamma}\right) t_{l-1},} \\
\left(1 \otimes \vec{\partial}_{-\gamma}\right) t_{l}=\left(e_{-\gamma} \otimes 1\right) t_{l-1}, \quad t_{l}\left(1 \otimes \overleftarrow{\partial}_{-\gamma}\right)=t_{l-1}\left(e_{-\gamma} \otimes 1\right), \\
\stackrel{\leftarrow}{\left.t_{l} \otimes 1\right)=t_{l-1}\left(1 \otimes e_{\gamma}\right),} \quad\left(\vec{\partial}_{\gamma} \otimes 1\right) t_{l}=\left(1 \otimes e_{\gamma}\right) t_{l-1} .
\end{gathered}
$$

These relations are integrable (with $t_{1}=e_{-\alpha} \otimes e_{\alpha}$ ) and yield a unique standard R-matrix on $\mathscr{A}^{\prime}$. 


\section{§8. The Standard Classical r-matrix for Simple Lie Algebras}

We shall now specialize, by stages, until we arrive at simple quantum groups, where a limiting process relates the standard R-matrix to a classical r-matrix.

Suppose that

$$
\operatorname{Card}(N):=l<\infty \text {. }
$$

Suppose next that the ideal $I$ (generated by the constants of $\mathscr{A}$ ) is generated by a complete set of Serre relations; that is, for each pair $\alpha, \beta \in N, \alpha \neq \beta$, there is a smallest positive integer $k_{\alpha \beta}$ such that there is a relation in $\mathscr{A} / I$ of the form

$$
\sum_{m=0}^{k_{\alpha \beta}} Q_{m}^{(\alpha, \beta)}\left(e_{\alpha}\right)^{m} e_{\beta}\left(e_{\alpha}\right)^{k_{\alpha \beta}-m}=0
$$

with coefficients $Q_{m}^{(\alpha, \beta)}$ in the field. The left side, as an element of $\mathscr{A}^{+}$, is a constant, and the penultimate paragraph of Section 3 applies. In particular, the relation (3.20) becomes

$$
e^{\varphi(\alpha, \beta)+\varphi(\beta, \alpha)+\left(k_{\alpha \beta}-1\right) \varphi(\alpha, \alpha)}=1,
$$

and the coefficients are

$$
Q_{m}^{k}=(-)^{m} e^{m \varphi(\alpha, \beta)} q^{m(m-1) / 2}\left(\begin{array}{c}
k \\
m
\end{array}\right)_{q}, \quad q:=e^{\varphi(\alpha, \alpha)} .
$$

We specialize further by supposing that the exponent in (8.3) vanish,

$$
\varphi(\alpha, \beta)+\varphi(\beta, \alpha)=\left(1-k_{\alpha \beta}\right) \varphi(\alpha, \alpha), \quad \alpha \neq \beta
$$

The form $(\cdot, \cdot)$ defined by

$$
(\alpha, \beta)=\varphi(\alpha, \beta)+\varphi(\beta, \alpha)
$$

will be called the restricted Killing form, and the $l$-by-l matrix with components

$$
A_{\alpha \beta}:=1-k_{\alpha \beta}=\frac{(\alpha, \beta)+(\beta, \alpha)}{(\alpha, \alpha)}
$$

will be called the generalized Cartan matrix; note that it is symmetrizable. Finally, a suitable restriction on $\operatorname{Card}(M)$ brings us to quantized Kac-Moody algebras.

Let $\mathscr{A}_{c l}^{\prime}$ be the algebra obtained from $\mathscr{A}^{\prime}$ when the relations $(2.4)$ are replaced by 


$$
\left[e_{\alpha}, e_{-\beta}\right]=\delta_{\alpha}^{\beta}(\varphi(\alpha, \cdot)+\varphi(\cdot, \alpha))
$$

If $\mathscr{A}_{c l}^{\prime}$ is a Kac-Moody algebra of finite type, resp. affine type, then we may say that $\mathscr{A}^{\prime}$ is a quantized Kac-Moody algebra of finite type, resp. affine type. But because $\mathscr{A}^{\prime}$ cannot be recovered from $\mathscr{A}_{c l}^{\prime}$ an autonomous definition is preferable.

Definition 8. Let $\mathscr{A}^{\prime}$ be as above; that is, the quotient of an algebra $\mathscr{A}$ as per Definition 2.1, with parameters satisfying (8.3), by the ideal generated by the Serre relations (8.2). We shall say that $\mathscr{A}^{\prime}$ is a quantized Kac-Moody algebra of finite type if (i) $\operatorname{Card} M=\operatorname{Card} N=l<\infty$, and (ii) the (symmetrizable) generalized Cartan matrix

$$
A_{\alpha \beta}=(1-k)_{\alpha \beta}=\frac{\varphi(\alpha, \beta)+\varphi(\beta, \alpha)}{\varphi(\alpha, \alpha)}
$$

is positive definite with $A_{\alpha \beta} \in\{0,-1, \cdots\}, \alpha \neq \beta$. We shall say that $\mathscr{A}^{\prime}$ is a quantized Kac-Moody algebra of affine type if (i) $\operatorname{Card} M=2+\operatorname{Card} N<\infty$, and (ii) the generalized Cartan matrix is positive semi-definite with $A_{\alpha \beta} \in\{0,-1, \cdots\}, \alpha \neq \beta$, and all its principal minors are positive definite.

The remainder of this section deals with Kac-Moody algebras of finite type.

The semi-classical limit of $R$ is defined by replacing

$$
\begin{aligned}
& \varphi(\cdot, \cdot) \rightarrow \hbar \varphi(\cdot, \cdot), \\
& e_{\alpha} \rightarrow \kappa e_{\alpha}, \quad e_{-\alpha} \rightarrow \kappa^{\prime} e_{-\alpha}, \quad \kappa \kappa^{\prime}=\hbar, \quad \alpha \in N,
\end{aligned}
$$

and developing the exponentials to first order in $\hbar$. Then Eq. (2.4) becomes

$$
\begin{aligned}
{\left[e_{\alpha}, e-\beta\right] } & =\delta_{\alpha \beta}(\varphi(\alpha, \cdot)+\varphi(\cdot, \alpha)) \\
& =: \delta_{\alpha \beta} H_{(\alpha)} \varphi(\alpha, \alpha) .
\end{aligned}
$$

It follows from (8.11) and (2.3) that

$$
\left[H_{(\alpha)}, e_{\beta}\right]=A_{\alpha \beta} e_{\beta}, \quad \alpha, \beta \in N .
$$

The definition (8.7) of the generalized Cartan matrix implies that $A_{\alpha \alpha}=2, \alpha \in N$, that $A_{\alpha \beta} \in\{0,-1,-2, \cdots\}, \alpha \neq \beta$, and that $A_{\alpha \beta} \neq 0$ implies $A_{\beta \alpha} \neq 0$. Special cases are affine Lie algebras and simple Lie algebras. The latter are characterized by two additional properties of $\left(A_{\alpha \beta}\right)$ : indecomposability and $\operatorname{det}(A)>0$. We now assume that both hold, and that $\left\{H_{(\alpha)}, \alpha \in N\right\}$ generates $\mathscr{A}^{0}$. 
The (classical) r-matrix $r$ associated with the standard R-matrix (2.5) is defined by

$$
R=1+\hbar r+o\left(\hbar^{2}\right)
$$

Two terms in $r$ are obvious: $r=\varphi+\Sigma e_{-\alpha} \otimes e_{\alpha}+$ ?, with the sum extending over simple roots. Evaluating the remaining terms is more difficult, because a) we do not have a sufficiently explicit expression for the coefficients $t_{(\alpha)}^{\left(\alpha^{\prime}\right)}$ and b) because all these coefficients are singular in the classical limit. Both these difficulties are avoided by the recursion relation (7.1), as we shall see later. The result, which was known by indirect means, with a particular normalization of the non-simple roots, is that

$$
r=\varphi+\sum_{\alpha \in \Delta^{+}} E_{-\alpha} \otimes E_{\alpha},
$$

where $\Delta^{+}$is the set of positive roots. (Definition 9.) This is what we call the standard r-matrix for a simple Lie algebra. It satisfies the classical Yang-Baxter relation

$$
\left[r_{12}, r_{13}+r_{23}\right]+\left[r_{13}, r_{23}\right]=0
$$

and

$$
r+r^{t}=\hat{K}
$$

the Killing form of $\mathrm{g}$. In the list of (constant) r-matrices obtained by Belavin and Drinfeld [BD], (8.14) is the simplest. The quantum groups to which these r-matrices are associated are the twisted quantum groups of Reshetikhin and others $[\mathrm{R}][\mathrm{Sc}][\mathrm{Su}]$.

\section{§9. The Standard Classical r-matrix for Untwisted Loop Algebras}

A quantized affine Kac-Moody algebra can be described as follows. Let $\hat{\mathscr{A}}^{\prime}$ be as above, with parameters satisfying (1.8) and Serre relations (1.9), with root generators $\left\{e_{ \pm \alpha}\right\} \alpha=0, \cdots, l$ and Cartan generators $H_{1}, \cdots, H_{l}, c, d$, such that the subset that consists of $\left\{e_{ \pm \alpha}\right\} \alpha \neq 0$ and $H_{1}, \cdots, H_{l}$ generates a subalgebra $\mathscr{A}^{\prime}$ that is a quantized Kac-Moody algebra of finite type. Let $\hat{\varphi}$ refer to $\hat{\mathscr{A}}^{\prime}$ and $\varphi$ to $\mathscr{A}^{\prime}$, and suppose that

$$
\hat{\varphi}=\varphi+u c \otimes d+(1-u) d \otimes c, \quad\left[d, e_{ \pm \alpha}\right]= \pm \delta_{\alpha}^{0} e_{ \pm 0} .
$$


with some $u \in C$. Suppose that $c$ is central and that the extra root defined by $\left[H_{a}, e_{0}\right]=H_{a}(0) e_{0}$ is such as to make the generalized Cartan matrix of $\hat{\mathscr{A}}^{\prime}$ positive semi-definite with all its principal minors positive. Then $\hat{\mathscr{A}}^{\prime}$ is a quantized affine Kac-Moody algebra.

Consider a quantized affine Kac-Moody algebra $\hat{\mathscr{A}}^{\prime}$, with generators $e_{ \pm 0}, \cdots, e_{ \pm l}$ and $H_{1}, \cdots, H_{l}, c, d$. Renormalize as in (8.10) and pass to the classical limit.

Definition 9. Positive root vectors are elements in $\mathscr{A}_{c l}^{\prime+}$ defined recursively. (a) The generators $e_{\alpha}$ are positive root vectors. (b) If $E_{i}$ and $E_{j}$ are positive root vectors and $\left[E_{i}, E_{j}\right] \neq 0$, then $\left[E_{i}, E_{j}\right]$ is a positive root vector. (c) All positive root vectors are obtained in this way from the generators. Negative root vectors are in $\mathscr{A}_{c l}^{\prime}$ and are defined analogously.

Let $\left\{E_{i}\right\} i=1, \cdots, n,+$ be the positive root vectors, labelled in such a way that

$$
\left[e_{\alpha}, E_{+}\right]=0=\left[e_{-\alpha}, E_{-}\right]
$$

and

$$
\left[E_{i}, E_{-}\right] \in \mathscr{A}_{c l}^{\prime 0} \cdot \mathscr{A}_{c l}^{\prime-}, \quad\left[E_{-i}, E_{+}\right] \in \mathscr{A}_{c l}^{\prime 0} \cdot \mathscr{A}_{c l}^{\prime+}
$$

Then we may refer to $E_{+}$as a highest root vector.

Suppose that the extra root $H_{a}(0)=H_{a}\left(E_{-}\right)$, and pass to the associated untwisted loop algebra $C\left[\lambda, \lambda^{-1}\right] \otimes \mathscr{A}_{c l}^{\prime}$ by substituting

$$
\hat{\varphi} \rightarrow \varphi, \quad e_{0}:=\lambda E_{-}, \quad e_{-0}:=\lambda^{-1} E_{+} .
$$

(Replacing $\hat{\varphi}$ by $\varphi$ amounts to taking the quotient by the ideal generated by the central element $c$.)

After the renormalization (8.10) $t_{n}$ is of order $\hbar$ and the classical r-matrix is defined by (8.13),

$$
R=1+\hbar r+o\left(\hbar^{2}\right)
$$

The Yang-Baxter relation for $R$ is equivalent to the recursion relation (7.1),

$$
\left[t_{n}, 1 \otimes e_{-\gamma}\right]=\left(e_{-\gamma} \otimes e^{\varphi(\gamma, \cdot)}\right) t_{n-1}-t_{n-1}\left(e_{-\gamma} \otimes e^{-\varphi(\cdot, \gamma)}\right), \quad n \geq 1 .
$$

To lowest order in $\hbar$ this becomes

$$
\left[t_{1}, 1 \otimes e_{-\gamma}\right]=e_{-\gamma} \otimes\left(\varphi+\varphi^{t}\right)(\gamma),
$$




$$
\left[t_{n}, 1 \otimes e_{-\gamma}\right]=\left[e_{-\gamma} \otimes 1, t_{n-1}\right], \quad n \geq 2,
$$

which is the same as

$$
\left[1 \otimes e_{-\gamma}+e_{-\gamma} \otimes 1, r-\varphi\right]+\left[t_{1}, 1 \otimes e_{-\gamma}\right]=0, \quad \gamma=0, \cdots, l,
$$

with $t_{1}=\Sigma e_{-\alpha} \otimes e_{\alpha}$, or

$$
\left[1 \otimes e_{-\gamma}+e_{-\gamma} \otimes 1, r\right]=\varphi(\cdot, \gamma) \wedge e_{-\gamma}
$$

This result is just the classical limit of the relation $\Delta\left(e_{-\gamma}\right) R=R \Delta^{\prime}\left(e_{-\gamma}\right)$, which explains why it determines $r$.

We normalize the root vectors so that the Casimir element takes the form

$$
C=\varphi+\varphi^{t}+\sum E_{-i} \otimes E_{i}+\sum E_{i} \otimes E_{-i}
$$

Then

$$
\begin{aligned}
& {\left[e_{-\gamma}, E_{-i}\right]=c E_{-j} \text { implies that }\left[E_{j}, e_{-\gamma}\right]=c E_{i}, \quad \gamma \neq 0,} \\
& {\left[e_{-0}, E_{-i}\right]=c E_{j} \text { implies that }\left[E_{-j}, e_{-0}\right]=c E_{i}, \quad \gamma \neq 0,}
\end{aligned}
$$

It may be seen from the structure of $t_{n}$ that it is a polynomial of order $n$ in $\lambda / \mu$. The recursion relation shows that the classical limit is in $\mathscr{A}_{c l}^{\prime} \otimes \mathscr{A}_{c l}^{\prime}$. The classical r-matrix can therefore be expressed as a formal power series in $x=\lambda / \mu$,

$$
r=\varphi+\psi(x)^{a b} H_{a} \otimes H_{b}+\sum f_{i}(x) E_{-i} \otimes E_{i}+\sum g_{i}(x) E_{i} \otimes E_{-i}
$$

Now it is easy to work out the implications of Eq. (9.7), namely, first taking $\gamma \neq 0$,

$$
\begin{aligned}
0= & {\left[1 \otimes e_{-\gamma}+e_{-\gamma} \otimes 1, \psi(x)^{a b} H_{a} \otimes H_{b}+\sum f_{i}(x) E_{-i} \otimes E_{i}\right.} \\
& \left.+\sum g_{i}(x) E_{i} \otimes E_{-i}\right]+\sum e_{-\alpha} \otimes\left[e_{\alpha}, e_{-\gamma}\right] \\
= & e_{-\gamma} \otimes\left(\psi(\gamma, \cdot)+\left(1-f_{\gamma}\right)\left(\varphi+\varphi^{t}\right)(\gamma)\right) \\
& +\left(\psi(\cdot, \gamma)-g_{\gamma}\left(\varphi+\varphi^{t}\right)(\gamma)\right) \otimes e_{-\gamma} \\
& +\sum f_{i}\left[e_{-\gamma}, E_{-i}\right] \otimes E_{i}+\sum^{\prime} f_{i} E_{-i} \otimes\left[e_{-\gamma}, E_{i}\right] \\
& +\sum^{\prime} g_{i}\left[e_{-\gamma}, E_{i}\right] \otimes E_{-i}+\sum g_{i} E_{i} \otimes\left[e_{-\gamma}, E_{-i}\right], \quad \gamma \neq 0 .
\end{aligned}
$$

The prime on $\Sigma^{\prime}$ means that the summation is over roots that are not simple. Cancellation in the last two lines imply, in view of (9.9) and since the adjoint action is irreducible, that $f_{i}=f, g_{i}=g, i=1, \cdots, l$. Cancellation in the two first lines now tells us that $\psi \propto \varphi+\varphi^{t}$, hence $\psi$ is symmetric, and it 
follows that $g=f-1$. This gives us

$$
r=\varphi+\sum E_{-i} \otimes E_{i}+g(x) C,
$$

which is actually obvious: The two first terms is a special solution and the last term is the only thing that commutes with $\Delta_{0}\left(e_{-\gamma}\right)=1 \otimes e_{-\gamma}+e_{-\gamma} \otimes 1$. Next, Eq. (9.7) with $\gamma=0$,

$$
\begin{aligned}
0= & {\left[1 \otimes e_{-0}+e_{-0} \otimes 1, \psi(x)^{a b} H_{a} \otimes H_{b}+\sum f_{i}(x) E_{-i} \otimes E_{i}\right.} \\
& \left.+\sum g_{i}(x) E_{i} \otimes E_{-i}\right]+\sum e_{-\alpha} \otimes\left[e_{\alpha}, e_{-0}\right] \\
= & E_{+} \otimes\left(\frac{1}{\mu} \psi(0, \cdot)+\left(\frac{1}{\mu}-\frac{g}{\lambda}\right)\left(\varphi+\varphi^{t}\right)(0)\right) \\
& +\left(\frac{1}{\lambda} \psi(\cdot, 0)-\frac{f}{\mu}\left(\varphi+\varphi^{t}\right)(0)\right) \otimes E_{+} \\
& +\frac{f}{\mu_{i \neq+}} \sum\left[E_{+}, E_{-i}\right] \otimes E_{i}+\frac{g}{\lambda} \sum_{i \neq+} E_{i} \otimes\left[E_{+}, E_{-i}\right] .
\end{aligned}
$$

This yields $g=x f$ and the result is that

$$
r=\varphi+\sum E_{-i} \otimes E_{i}+\frac{x}{1-x} C, \quad x=\lambda / \mu
$$

which agrees with the simplest $r$-matrix in $[\mathrm{BD}]$, but in the notation of $[\mathrm{J}]$.

\section{§10. The Standard Classical r-matrix for Twisted Loop Algebras}

The construction of a twisted affine Kac-Moody algebra [K] involves two simple Lie algebras, $\mathfrak{g}$ and a subalgebra $\mathfrak{g}_{0}$, such that $\mathfrak{g}$ admits a diagram automorphism of order $k=2$ or 3 to which is associated a Lie algebra automorphism $\mu$ that centralizes $\mathfrak{g}_{0}$. The eigenvalues of $\mu$ are of the form $\omega^{j}, j=0,1, \cdots$, and $\mathfrak{g}=\Sigma_{j=0}^{k-1} \mathfrak{g}_{j}$, where $\mathfrak{g}_{j}$ is the sum of the eigenspaces with eigenvalues $\omega^{j \bmod k}$. The restriction of the adjoint action of $\mathfrak{g}$ to $\mathfrak{g}_{0}$ acts irreducibly on each $\mathfrak{g}_{j}$.

Now let $\left\{H_{a}, e_{ \pm \alpha}\right\} \alpha=1, \cdots, n$ be a Chevalley basis for $g_{0}$, and let $E_{+}$be a highest weight vector (for the action of $g_{0}$ ) in $g_{1}$. Then $\left\{e_{\alpha}\right\}, E_{-}$generate $g$, and

$$
\left[e_{\alpha}, E_{+}\right]=0=\left[e_{-\alpha}, E_{-}\right] \text {. }
$$

The twisted loop algebra $\hat{g}=C\left[\lambda, \frac{1}{\lambda}\right] \otimes g$ is generated by $\left\{e_{ \pm \alpha}\right\}, \alpha=0, \cdots, n$, 
with

$$
e_{0}=\lambda E_{-}, \quad e_{-0}=\frac{1}{\lambda} E_{+} .
$$

This algebra is of the type $\mathscr{A}_{c l}^{\prime}$, so our standard R-matrix applies. We define $r$ in terms of the expansion of $R$ in powers of $\hbar$ and work out the implications of the relations $(9.7)$.

Let $\left\{E_{i}\right\}$ be a Weyl basis for $g_{0}$ and normalize so that the Casimir element for that algebra is

$$
C_{0}=\varphi+\varphi^{t}+\sum E_{-i} \otimes E_{i}+\sum E_{i} \otimes E_{-i}
$$

Then a special solution of (9.7) with $\gamma \neq 0$ is given by the first two terms in (9.13) and the general solution is

$$
r=\varphi+\sum E_{-i} \otimes E_{i}+\sum_{0}^{k-1} f_{j} C_{j}
$$

where $C_{j}$ is the projection of the Casimir element $C$ of $\mathfrak{g}$ on $\mathfrak{g}_{j}$, on the first factor. Now (9.7), with $\gamma=0$ :

$$
\begin{aligned}
& 0= {\left[1 \otimes e_{-0}+e_{-0} \otimes 1, \sum E_{-i} \otimes E_{i}+\sum f_{j} C_{j}\right]+\sum e_{-\alpha} \otimes\left[e_{\alpha}, e_{-0}\right] } \\
&=\frac{1}{\mu} \sum\left[E_{+}, E_{-i}\right] \otimes E_{i}+\sum f_{j}\left(\frac{1}{\lambda}\left[1 \otimes E_{+}, C_{j}\right]+\frac{1}{\mu}\left[E_{+} \otimes 1, C_{j}\right]\right)+\frac{1}{\mu} E_{+} \otimes\left(\varphi+\varphi^{t}\right)(0) \\
&=\frac{1}{\mu}\left[E_{+} \otimes 1, C_{0}\right]+\sum\left(\frac{f_{j}}{\lambda}\left[1 \otimes E_{+}, C_{j}\right]+\frac{f_{j-1}}{\mu}\left[E_{+} \otimes 1, C_{j-1}\right] .\right.
\end{aligned}
$$

This vanishes iff

$$
\begin{aligned}
& f_{1}=x\left(f_{0}+1\right), \quad f_{0}=x f_{1}, \quad k=2, \\
& f_{1}=x\left(f_{0}+1\right), \quad f_{2}=x f_{1}, \quad f_{0}=x f_{2}, \quad k=3,
\end{aligned}
$$

That is,

$$
f_{j}=\frac{x^{j}}{1-x^{k}} C_{j}-\delta_{j}^{0} C_{0}
$$

Finally, the unique solution is 


$$
r=\varphi+\sum E_{-i} \otimes E_{i}-C_{0}+\frac{1}{1-x^{k}} \sum_{0}^{k-1} x^{j} C_{j},
$$

again in agreement with $[\mathrm{BD}]$, in the notation of $[\mathrm{J}]$.

Remark. Choose a basis of weight vectors in $\mathfrak{g}_{1}$, then

$$
C_{1}=E_{-} \otimes E_{+}+E_{+} \otimes E_{-}+\cdots
$$

with unit coefficients for the contributions with highest weight. This follows from the normalization in (10.3) and fact that $1 \otimes E_{+}+E_{+} \otimes 1$ commutes with $C=\Sigma C_{j}$.

\section{§11. Including the Central Extension}

The untwisted case. The extension is recovered by omitting the replacement of $\hat{\varphi}$ by $\varphi$ in (9.3). We can still represent the r-matrix as a power series in $x=\lambda / \mu$, but it is no longer true, as it was in the case of the loop group, that $\left[e_{0}, e_{-0}\right]=\left[E_{-}, E_{+}\right]$. Instead,

$$
\left[e_{0}, e_{-0}\right]=\left(\hat{\varphi}+\hat{\varphi}^{t}\right)(0)=\left[E_{-}, E_{+}\right]+c .
$$

More generally, for polynomials $f, g \in C\left[\lambda, \frac{1}{\lambda}\right]$, and $x, y \in \mathscr{A}_{c l}^{\prime}$,

$$
[f x, g y]=f g[x, y]+c\langle x, y\rangle \operatorname{Res}\left(f^{\prime} g\right),
$$

where the form $\langle$,$\rangle is the invariant form on \mathscr{A}_{c l}^{\prime}$ normalized as follows: If the Casimir element is $C^{i j} x_{i} \otimes x_{j}$, then $\left\langle x_{i}, x_{j}\right\rangle=\left(C^{-1}\right)_{i j} ; \operatorname{Res}(f)$ is the constant term in $\lambda f$.

Remark. This normalization implies that

$$
\left[f C_{12}, g C_{23}\right]=f g\left[C_{12}, C_{23}\right]+c_{2} C_{13} \operatorname{Res}\left(f^{\prime} g\right) \text {. }
$$

This change leaves (9.12) and (9.13) unaffected, while (9.14) becomes

$$
\begin{aligned}
0= & E_{+} \otimes\left(\frac{1}{\mu} \psi(0, \cdot)+\frac{1}{\mu}\left(\hat{\varphi}+\hat{\varphi}^{t}\right)(0)+\left[e_{0}, g(x) E_{-}\right]\right) \\
& +\left(\frac{1}{\lambda} \psi(\cdot, 0)-\frac{f}{\mu}\left(\varphi+\varphi^{t}\right)(0)\right) \otimes E_{+} \\
& +\frac{f}{\mu_{i \neq+}}\left[E_{+}, E_{-i}\right] \otimes E_{i}+\frac{g}{\lambda} \sum_{i \neq+} E_{i} \otimes\left[E_{+}, E_{-i}\right] .
\end{aligned}
$$


The modification in the second term ( $\varphi$ replaced by $\hat{\varphi})$ is exactly compensated by a new contribution from the linear $\lambda$-term in $g$. (There is no linear $\mu$-term in $f$ ). The conclusion is that the new r-matrix is

$$
\hat{r}=\hat{\varphi}+\sum E_{-i} \otimes E_{i}+\frac{x}{1-x} C
$$

The twisted case. It is easy to verify, with the help of the remark at the end of Section 3, that the restitution $\varphi \rightarrow \hat{\varphi}$ can be made without affecting the cancellations; so the result is that

$$
\hat{r}=\hat{\varphi}+\sum E_{-i} \otimes E_{i}-C_{0}+\frac{1}{1-x^{k}} \sum x^{j} C_{j}
$$

It is amusing to verify directly that the classical Yang Baxter relation for $r$,

$$
Y B(r):=\left[r_{12}, r_{13}+r_{23}\right]+\left[r_{13}, r_{23}\right]=0,
$$

implies the same relation for $\hat{r}$ : The inclusion of the extra term in $\hat{\varphi}$ means that

$$
Y B(\hat{r})=Y B(r)+\left[r_{13},(c \otimes d)_{23}\right] .
$$

The evaluation of $Y B(r)$ now has to take into account the new term (involving c) in Eq.(11.2). Actually, only $\left[r_{12}, r_{23}\right]$ is affected, and with the aid of Eq.(11.3) one finds that the new contribution is

$$
Y B(r)=c_{2} \lambda \frac{d}{d \lambda} r_{13},
$$

which exactly cancels the other term. In the twisted case one must use the following generalization of Eq.(11.3):

$$
\left[f C_{j 12}, g C_{j^{\prime} 23}\right]=f g\left[C_{j 12}, C_{j^{\prime} 23}\right]+\delta_{j}^{i^{\prime}} c_{2} C_{j 13} \operatorname{Res}\left(f^{\prime} g\right) .
$$

\section{DEFORMATIONS}

\section{§12. First Order Deformations}

Quantum groups can be understood as deformations of the Hopf structure associated with Lie algebras or Kac-Moody algebras. The point of view that 
emphasizes the direct connection between quantum groups and Lie groups, as well as the deep roots of quantum groups in deformation theory and in the theory of *-products, has been shown to lead to profound insight into their general structure $[\mathrm{BFGP}][\mathrm{BP}][\mathrm{EK}]$. Here we use deformation theory with a different purpose. The initial structure is the bialgebra associated with a standard R-matrix, with a fixed set of parameters. The deformed structure is a bialgebra equipped with an R-matrix that is non-standard and that does not commute with the Cartan sub-algebra. We emphasize that the context is more general than quantized Kac-Moody algebras.

This work was initiated with the aim of calculating the universal R-matrices associated with simple Lie algebras, as deformations of the standard universal R-matrix. We shall establish a direct correspondence between the classical r-matrices of Belavin and Drinfeld on the one hand, and the deformations of the standard, universal R-matrix for simple quantum groups on the other. In preparation for this we have explored the meaning of the Yang-Baxter relation in a much more general context, and we shall endeavor to maintain this generality in our approach to deformations. But, as for the types of deformations, we shall limit our study in a way that seems natural in the context of quantum groups.

A deformation of the standard R-matrix is a formal series

$$
R_{\epsilon}=R+\epsilon R_{1}+\epsilon^{2} R_{2}+\cdots
$$

Here $R$ is a standard R-matrix on $\mathscr{A}^{\prime}=\mathscr{A} / I$ with any choice of parameters and the ideal $I$ determined by them. The coefficients $t_{(\alpha)}^{\left(\alpha^{\prime}\right)}$ of $R$ are determined by the Yang-Baxter relation, and we attempt to find $R_{1}, R_{2}, \cdots$ so that $R_{\epsilon}$ will satisfy the same relation to each order in $\epsilon$. To make this program precise, we must specify the nature of the leading term; the remainder should then be more or less unique.

Recall that $R$ "commutes with Cartan." An element $Q \in \mathscr{A} \otimes \mathscr{A}$ is said to have weight $w$ if

$$
\left[H_{a} \otimes 1+1 \otimes H_{a}, Q\right]=w_{a} Q, \quad w_{a} \in C, \quad a \in M .
$$

Thus $R$ has weight zero. The image of $Q$ by the projection $\mathscr{A} \otimes \mathscr{A} \rightarrow \mathscr{A}^{\prime} \otimes \mathscr{A}^{\prime}$ has the same weight. We shall suppose that $R_{1}$ is homogeneous (has weight), but this restriction is inessential and will be relaxed later.

Recall further that $R$ is driven by the linear term; by virtue of the Yang-Baxter relation, $R$ is completely determined by the term $e_{-\alpha} \otimes e_{\alpha}$. It is 
natural to study deformations that are driven by a similar term, with fixed, non-zero weight:

$$
R_{1}=S\left(e_{ \pm \sigma} \otimes e_{ \pm \rho}\right)+\cdots
$$

with $\sigma, \rho$ fixed and the factor $S$ is in $\mathscr{A}^{0}$. (The unwritten terms are of higher order, in a sense that we shall make precise in a moment.) Such deformations may be called "non-singular", to contrast them to singular deformations for which the term of order $\epsilon$ is either absent or else of a form that sets it appart from the driving term in the undeformed R-matrix. We do not claim that this exhausts the possibilities. In fact, we know of a "singular" deformation that is driven by an $R_{1}$ of higher order in the generators [FG2]. It is highly special and occurs only when some of the parameters are roots of unity. But we believe that the deformations studied here have the best chance of possessing a cohomological interpretation.

We shall now make precise the concept of "higher order".

Proposition 12. The algebra $\mathscr{A}^{\prime}=\mathscr{A} / I$ is $Z$-graded, with grade $e_{ \pm \alpha}= \pm 1$, grade $H_{a}=0 . \quad$ An alternative grading is obtained by reversing the sign.

Proof. This is a consequence of the fact that the generators of $I$ are homogeneous; $\mathscr{A}^{\prime}$ inherits the grading of $\mathscr{A}$.

The standard R-matrix is a formal series $\Sigma_{k} \psi_{k}^{-} \otimes \psi_{k}^{+}, \psi_{k}^{ \pm} \in \mathscr{A}^{\prime}$. We use the grading of Proposition 12 in the second space, the alternative grading in the first space; then grade $\psi_{k}^{+}=k$ and $R$ is a formal sum of terms with grade $(k, k), k=0,1,2, \cdots$. This grading is an extension of that used previously, made necessary by the appearance of $e_{\sigma}$ in the first space and $e_{-\rho}$ in the second.

With the inclusion of $(12.3)$ the grades descend to $(-1,-1)$. Finally, the unwritten terms in (12.3) is a series by ascending grades. The fact that the grades are bounded below is fundamental. We claim that $R_{\epsilon}$, a formal series in $\epsilon$, each term a formal series in ascending grades, if it satisfies the Yang-Baxter relation, is completely determined by the choice of the two generators $e_{ \pm \sigma}$ and $e_{ \pm \rho}$ in (12.3).

We shall see that the standard R-matrix on $\mathscr{A}^{\prime}$, with the parameters of $\mathscr{A}^{\prime}$ in general position, is rigid with respect to deformations of the type (12.3). We begin our investigation by establishing some conditions on the parameters that are necessary for the existence of a deformation. We shall study each of the 
four possibilities envisaged by (12.3) separately. We organize the contributions to

$$
Y B_{\ell}:=R_{\ell 12} R_{\ell 13} R_{\ell 23}-R_{\ell 23} R_{\epsilon 13} R_{\ell 12}
$$

in the same way as the contributions to $Y B$. A term $\psi_{1} \otimes \psi_{2} \otimes \psi_{3}$ is said to have grade $(l, n)$ if $\psi_{3}$ has grade $n$ and $\psi_{1}$ has alternative grade $l$. We limit ourselves to terms linear in $\epsilon$ and end this section by disposing of three of the four possibilities in (12.3).

Deformations of Types $e_{-\sigma} \otimes e_{\rho}, e_{\sigma} \otimes e_{\rho}$ and $e_{-\sigma} \otimes e_{-\rho}$. Suppose first that the driving term in $R_{1}$ is

$$
S\left(e_{-\sigma} \otimes e_{\rho}\right), \quad S \in \mathscr{A}^{0} \otimes \mathscr{A}^{0} .
$$

We examine the contributions to $Y B_{\varepsilon}$ of order $\epsilon$.

The lowest grades are $(1,0)$ and $(0,1)$, with contributions

$$
\begin{aligned}
& \left(S e_{-\sigma} \otimes e_{\rho}\right)_{12} R_{13}^{0} R_{23}^{0}-R_{23}^{0} R_{13}^{0}\left(S e_{-\sigma} \otimes e_{\rho}\right)_{12}, \\
& R_{12}^{0} R_{13}^{0}\left(S e_{-\sigma} \otimes e_{\rho}\right)_{23}-\left(S e_{-\sigma} \otimes e_{\rho}\right)_{23} R_{13}^{0} R_{12}^{0},
\end{aligned}
$$

respectively. These vanish if and only if

$$
e^{\varphi(\sigma, \cdot)-\varphi(\rho, \cdot)}=1=e^{\varphi(\cdot, \sigma)-\varphi(\cdot, \rho)} .
$$

In grade $(1,1)$ we encounter additional restrictions,

$$
e^{\varphi(\rho, \cdot)+\varphi(\cdot, \sigma)}=1
$$

Conditions (12.4)-(12.5) are necessary. It follows that

$$
e^{\varphi(\rho, \alpha)+\varphi(\alpha, \rho)}=1=e^{\varphi(\sigma, \alpha)+\varphi(\alpha, \sigma)}, \quad \alpha \in N .
$$

These are conditions that are familiar from our investigation of constants, see Eq.(3.8). The relations that are thus implied are

$$
e_{\rho} e_{\alpha}-e^{\varphi(\rho, \alpha)} e_{\alpha} e_{\rho}=0=e_{\sigma} e_{\alpha}-e^{\varphi(\sigma, \alpha)} e_{\alpha} e_{\sigma}, \quad \forall \alpha \in N
$$

This constitutes a high degree of commutativity in $\mathscr{A}^{\prime}$ and takes us far away from our main interest in simple quantum groups. We therefore end our investigation of the type $e_{-\sigma} \otimes e_{\rho}$ at this point.

Similar results are obtained for deformations of type $e_{-\sigma} \otimes e_{-\rho}$ and 
$e_{\sigma} \otimes e_{\rho}$

\section{§13. First Order Deformations of Type $e_{\sigma} \otimes e_{-\rho}$ and the Classical Limit}

We come to the last case envisaged in $\S 12$. Eq. (12.3), when the driving term in $R_{1}$ has the form

$$
S\left(e_{\sigma} \otimes e_{-\rho}\right), \quad S \in \mathscr{A}^{0} \otimes \mathscr{A}^{0} .
$$

This term has grade $(-1,-1)$; it is the only term in $R_{1}$ with this grade, the lowest. The factor $S$, and all other terms in $R_{1}$, are completely determined by the Yang-Baxter relation $Y B_{\epsilon}=0$ to first order in $\epsilon$. Besides (13.1) there is in $R_{1}$ one other term with only two roots, of the form

$$
S^{\prime}\left(e_{-\rho} \otimes e_{\sigma}\right), \quad S^{\prime} \in \mathscr{A}^{0} \otimes \mathscr{A}^{0}
$$

it has grade $(1,1)$.

Theorem 13.1. Let $R$ be the standard $R$-matrix described in Theorem 7. Suppose that $R+\epsilon R_{1}$ is a first order deformation, satisfying the Yang-Baxter relation to first order in $\epsilon$. Suppose also that the term of lowest grade in $R_{1}$ has the form (13.1); then the parameters satisfy

$$
e^{\varphi(\cdot, \rho)+\varphi(\sigma \cdot)}=1 .
$$

Conversely, when the parameters are in general position on this surface, then there exists a unique first order deformation such that the term of lowest grade has the form (13.1), namely

$$
R_{1}=R\left(K e_{\sigma} \otimes K e_{-\rho}\right)-\left(K e_{-\rho} \otimes K e_{\sigma}\right) R,
$$

with $K:=e^{\varphi(\cdot, \rho)}$.

Proof. An easy calculation in the lowest grades shows that (13.3) is necessary and that $S=K \otimes K$, up to a numerical factor that we fix once and for all.

Let $R_{1}^{i}, i=1,2$, be the two summands in (13.4). The term of order $\epsilon$ in $Y B_{\epsilon}$ is the sum of the following six quantities:

$$
\begin{aligned}
& A_{12}^{i}=\left(R_{1}^{i}\right)_{12} R_{13} R_{23}-R_{23} R_{13}\left(R_{1}^{i}\right)_{12}, \\
& A_{13}^{i}=R_{12}\left(R_{1}^{i}\right)_{13} R_{23}-R_{23}\left(R_{13}^{i}\right)_{13} R_{23}, \\
& A_{23}^{i}=R_{12} R_{13}\left(R_{1}^{i}\right)_{23}-\left(R_{1}^{i}\right)_{23} R_{13} R_{12}, \quad i=1,2 .
\end{aligned}
$$


Step 1. We begin with the term that contains the lowest grade, $(1,1)$ :

$$
\begin{aligned}
& A_{13}^{1}=R^{i}[-\alpha] R^{j}[-\beta] K e_{\sigma} \otimes R_{i}[\alpha] R^{k}[-\gamma] \otimes R_{j}[\beta] K e_{-\rho} R_{k}[\gamma]-\cdots, \\
& {[-\alpha] \otimes[\alpha]:=e_{-\alpha_{1}} \cdots e_{-\alpha_{l}} t_{(\alpha)}^{\left(\alpha^{\prime}\right)} e_{\alpha_{1}^{\prime}} \cdots e_{\alpha_{l}} .}
\end{aligned}
$$

A sum over indices and numbers of indices ( $l \alpha$ 's, $m \beta$ 's and $n \gamma$ 's) is understood, and $-\cdots$ stands for the reflected term. Using the fact that $R$ satisfies $Y B=0$ we can convert (13.6) to

$$
A_{13}^{1}=R^{i}[-\alpha] R^{j}[-\beta] K e_{\sigma} \otimes R_{i}[\alpha] R^{k} K[-\gamma] \otimes R_{j}[\beta] K R_{k}\left[e_{-\rho},[\gamma]\right]+\cdots,
$$

where $+\cdots$ stands for a similar expression that contains a factor $\left[e_{\sigma},[-\alpha]\right]$ in the first space. We have used (13.3) and continue to use this relation without comment.

Step 2. Evaluate the commutators in (13.7) using (5.1) and (5.7). The result

$$
A_{13}^{1}=R^{i}[-\alpha] R^{j}[-\beta] K e_{\sigma} \otimes R_{i}[\alpha] R^{k} K[-\gamma] e_{-\rho} \otimes R_{j}[\beta] K R_{k}[\gamma] K^{-1}+\cdots
$$

is a sum of four similar expressions. Note that the evaluation of the commutators involves a shift in the summation indices $l, m, n$. The generators $e_{\sigma}, e_{-\rho}$, in spaces 1,3 in (13.7), are now in spaces 1 and 2 , and the lowest grades in $(13.8)$ are $(-1,0)$ and $(0,-1)$.

Step 3. Now write down the full expression for $A_{12}^{1}+A_{23}^{1}$; it also contains four similar terms. Two of them cancel two of the terms in (13.8), by virtue of the relation $Y B=0$.

Step 4. Combine the remaining two terms from (13.8) with the remaining two terms from $A_{12}^{1}+A_{23}^{1}$ and verify that

$$
\begin{aligned}
& A_{12}^{1}+A_{13}^{1}+A_{23}^{1} \\
= & R^{i}[-\alpha] K R^{j}\left[e_{\sigma},[-\beta]\right] \otimes R_{i}[\alpha] K R^{k} e_{-\rho}[-\gamma] \otimes R_{j} K[\beta] R_{k} e^{\varphi(\rho, \cdot)}[\gamma]+\cdots,
\end{aligned}
$$

where $+\cdots$ stands for a term that contains a factor $\left[[\beta], e_{-}\right]$in the third space.

Step 5. Evaluate the commutators (second shift of summation indices) 


$$
\begin{aligned}
& =R^{i}[-\alpha] R^{j} K[-\beta] K^{-1} \otimes R^{i}[\alpha] K R^{k} e-\rho[-\gamma] \otimes R_{j} K[\beta] e_{\sigma} R_{k} e^{\varphi(\rho, \cdot)}[\gamma]+\cdots \\
& =: X_{1}+X_{2}+Y_{1}+Y_{2} .
\end{aligned}
$$

The lowest grades are now $(1,0)$ and $(0,1)$. The generator $e_{\sigma}$ has completed its journey towards the east and is found in the third space; the generator $e_{-\rho}$, travelling westward, is in space two.

Step 6. Two of the four terms in (13.10) are:

$$
\begin{aligned}
& X_{1}=R_{12} R_{13}\left\{\left(K e_{-\rho} \otimes K e_{\sigma}\right) R\right\}_{23}, \\
& Y_{2}=-R_{23} R_{13}\left\{\left(K e_{-\rho} \otimes K e_{\sigma}\right) R\right\}_{12} .
\end{aligned}
$$

Now add $A_{12}^{2}+A_{23}^{2}$ to (13.10) to get

$$
\begin{aligned}
& A_{12}^{1}+A_{13}^{1}+A_{23}^{1}+A_{12}^{2}+A_{23}^{2} \\
= & \tilde{X}_{1}+X_{2}+Y_{1}+\tilde{Y}_{2},
\end{aligned}
$$

where $\tilde{X}_{1}$ and $\tilde{Y}_{2}$ are obtained from $X_{1}$ and $Y_{2}$ by adding $A_{23}^{2}$ and $A_{12}^{2}$.

Step 7. Use the relation $Y B=0$ to modify the expressions for $\tilde{X}_{1}$ and $\tilde{Y}_{2}$; then notice that the four terms in (13.12) can be combined to two,

$$
=R^{i} K[-\alpha] R^{j}[-\beta] \otimes R_{i} K\left[e_{-\rho},[\alpha]\right] R^{k}[-\gamma] \otimes K e_{\sigma} R_{j}[\beta] R_{k}[\gamma]+\cdots,
$$

where the other term has a factor $\left[[-\gamma], e_{\sigma}\right]$ in the second space.

Step 8. Evaluate the commutators (third shift of summation indices),

$$
=R^{i} K[-\alpha] e_{-\rho} R^{j}[-\beta] \otimes R_{j} K[\alpha] K^{-1} R^{k}[-\gamma] \otimes K e_{\sigma} R_{j}[\beta] R_{k}[\gamma]+\cdots
$$

This expression has four terms; the lowest grade is (1.1). The generators $e_{\sigma}$, $e_{-\rho}$ have reached their final destination, $e_{\sigma}$ is in space three and $e_{-\rho}$ is in space one. What remains can be compared with the last of the six contributions to $Y B_{t}$, namely $A_{13}^{2}$.

Step 9, 10. Two of the four terms in (13.14) cancel each other because $Y B=0$ and the remaining two terms add up to $-A_{13}^{2}$.

This completes the verification of the claim that (13.4) defines a first order deformation of $R$. To complete the proof of Theorem 13.1 we must show 
that this expression (13.4) is unique. This was done by complete mathematical induction. We omit the details but point out that the key to the induction process is visible in Steps 2, 5 and 8, where the summation indices are shifted. Theorem 13.1 is proved.

Let $\mathscr{P}$ be the collection of pairs $(\sigma, \rho) \in N \otimes N$ such that (13.3) holds; each distinct pair defines a first order deformation $R+\epsilon R_{1}^{\sigma . \rho}$ of $R$. Because these deformations are only first order they generate a linear space

$$
R_{1}=\sum_{\sigma, \rho \in \mathscr{P}} C_{\sigma, \rho} R_{1}^{\sigma, \rho}
$$

with coefficients in $C$. The dimension of this space of first order deformations is zero for parameters in general position. It remains zero, generically, when the parameters are such that the ideal $I$ generated by the constants is non-zero and $R$ is defined on $\mathscr{A} / I$. The exceptional points in the space of parameters, at which there are pairs $(\sigma, \rho)$ satisfying (13.3), are bifurcation points in the space of generalized quantum groups.

To any first order deformation of $R$, there corresponds a first order deformation of $r$,

$$
R_{\epsilon}=1+\hbar r_{\epsilon}+o\left(\hbar^{2}\right), \quad r_{\epsilon}=r+\epsilon r_{1}+o\left(\epsilon^{2}\right)
$$

Eqs. (13.4) and (13.15) give us

$$
r_{1}=\sum_{\sigma, \rho \in \mathscr{P}} C_{\sigma, \rho}\left(e_{\sigma} \wedge e_{-\rho}\right)
$$

where $\mathscr{P}$ is the set of pairs with the property

$$
\varphi(\rho, \cdot)+\varphi(\cdot, \sigma):=0
$$

The original work of Belavin and Drinfeld culminates in a list of constant $r$-matrices that is complete up to equivalence. Their results have recently been re-derived in terms of deformation theory and the associated cohomology.

Proposition 13. [F] Let $r$ be the standard $r$-matrix (8.14) for a simple Lie algebra $\mathrm{g}$. The space of essential, first order deformations of $r$, satisfying (8.15) and (8.16), is

$$
H^{2}\left(\mathscr{L}^{*}, C\right)=\left\{r_{1}=\sum_{\sigma, \rho \in \mathscr{P}} C_{\sigma, \rho} e_{\sigma} \wedge e_{-\rho}+\sum \tilde{C}^{a b} H_{a} \otimes H_{b}\right\}
$$


The exact deformations are of finite order and coincide with the r-matrices of $[B D]$.

The second, Cartan term is not "essential" in the present context; it represents the freedom to vary the parameters. We conclude that

Theorem 13.2. The first order deformations of the standard R-matrix described in Theorem 13.1, upon specialization to a simple quantum group, are in one-to-one correspondence, via (13.16), with the first order essential deformations of the associated standard r-matrix, modulo variations of the parameters.

One concludes that the class of deformations investigated in Section 13 is wide enough to encompass the quantization of all simple Lie bialgebras. We shall see that the affine Kac-Moody algebras are provided for also.

\section{§14. Hopf Structure}

It is of some interest to verify that the standard R-matrix, satisfying the Yang-Baxter relation, actually intertwines the coproduct of a Hopf algebra with its opposite.

Proposition 14.1. (a) There exists a unique homomorphism $\Delta: \mathscr{A} \rightarrow \mathscr{A} \otimes$ $\mathscr{A}$, such that

$$
\begin{aligned}
& \Delta\left(H_{a}\right)=H_{a} \otimes 1+1 \otimes H_{a}, \quad a \in M, \\
& \Delta\left(e_{\alpha}\right)=1 \otimes e_{\alpha}+e_{\alpha} \otimes e^{\varphi(\alpha, \cdot)}, \\
& \Delta\left(e_{-\alpha}\right)=e^{-\varphi(\cdot, \alpha)} \otimes e_{-\alpha}+e_{-\alpha} \otimes 1, \quad \alpha \in N .
\end{aligned}
$$

(b) If $I \subset \mathscr{A}$ is the ideal generated by the constants in $\mathscr{A}^{+}$and $\mathscr{A}^{-}$, and $\mathscr{A}^{\prime}=\mathscr{A} / I$, then $\Delta$ induces a unique homomorphism $\mathscr{A}^{\prime} \rightarrow \mathscr{A}^{\prime} \otimes \mathscr{A}^{\prime}$ that will also be denoted $\Delta$, so that (14.1) holds with $H_{a}$ and $e_{ \pm \alpha}$ being interpreted as generators of $\mathscr{A} / I$.

(c) Let $\Delta^{\prime}$ be the opposite coproduct on $\mathscr{A} / I$, and $R$ the standard $R$-matrix on $\mathscr{A} / I$ (satisfying Yang-Baxter), then $\Delta R=R \Delta^{\prime}$.

(d) The algebra $\mathscr{A}$ becomes a Hopf algebra when endowed with the counit $\mathscr{E}$ and the antipode $S$. The former is the unique homomorphism $\mathscr{A} \rightarrow C$ such that

$$
\mathscr{E}(1)=1, \quad \mathscr{E}\left(H_{a}\right)=0, \quad a \in M,
$$




$$
\mathscr{E}\left(e_{ \pm \alpha}\right)=0, \quad \alpha \in N
$$

The antipode is the unique anti-automorphism $S: \mathscr{A} \rightarrow \mathscr{A}$ such that

$$
\begin{aligned}
& S(1)=1, \quad S\left(H_{a}\right)=-H_{a}, \quad a \in M, \\
& S\left(e_{\alpha}\right)=-e_{\alpha} e^{-\varphi(\alpha, \cdot)}, \quad S\left(e_{-\alpha}\right)=-e^{\varphi(\cdot, \alpha)} e_{-\alpha}, \quad \alpha \in N .
\end{aligned}
$$

(e) The counit $\mathscr{E}$ and the antipode $S$ of $\mathscr{A}$ induce analogous structures on $\mathscr{A}^{\prime}=\mathscr{A} /$ I such that (14.3) holds on $\mathscr{A}^{\prime}$.

Proof. (a) The verification amounts to checking that $\Delta(\mathscr{A})$ has the relations of $\mathscr{A}$, in particular,

$$
\left[\Delta\left(e_{\alpha}\right), \Delta\left(e_{-\beta}\right)\right]=\delta_{\alpha}^{\beta} \Delta\left(\left[e_{\alpha}, e_{-\beta}\right]\right) .
$$

(b) The ideal $I$ is generated by elements $x \in \mathscr{A}^{+}$and $y \in \mathscr{A}^{-}$such that $\left[e_{-\alpha}, x\right]=0=\left[e_{\alpha}, y\right], \alpha \in N$. Since $\Delta: \mathscr{A} \rightarrow \mathscr{A} \otimes \mathscr{A}$ is a homomorphism, $\Delta$ induces a homomorphism $\mathscr{A} / I \rightarrow(\mathscr{A} \otimes \mathscr{A}) / \Delta(I)$. We must show that $\Delta(I) \subset I \otimes \mathscr{A}$ $+\mathscr{A} \otimes I$. Since $I$ is generated by elementary constants, it is enough to show that, for an elementary constant $C, \Delta(C) \subset I \otimes \mathscr{A}+\mathscr{A} \otimes I$. Let $C \in \mathscr{A}^{+}$be an elementary constant; then $\left[e_{-\alpha}, C\right]=0$ and thus $\left[\Delta\left(e_{-\alpha}\right), \Delta(C)\right]=0, \alpha \in N$. If $C$ is of order $n$ in the generators, (14.1) shows that

$$
\Delta C=1 \otimes C+P^{1} \otimes P_{n-1}+P^{2} \otimes P_{n-2}+\cdots+C \otimes P_{0},
$$

where $P^{k}$ and $P_{k}$ are homogeneous of order $k$ in the $e_{\alpha}$ 's. Because $C$ is an elementary constant-Definition 4.1 - there is no constant among the $P^{k}, P_{k}$, $n=1, \cdots, n-1 ;$ then $\left[\Delta\left(e_{-\alpha}\right), \Delta(C)\right]=0$ implies that $\Delta C=1 \otimes C+C \otimes P_{0}$ which indeed belongs to $I \otimes \mathscr{A}+\mathscr{A} \otimes I$; consequently $\Delta$ provides a map $\mathscr{A} / I \rightarrow \mathscr{A} / I$ $\otimes \mathscr{A} / I$.

(c) We use the abbreviation - compare (13.6), Definition 2.2 and Eq. (2.9)-

$$
\begin{aligned}
R= & t_{(\alpha)}^{\left(\alpha^{\prime}\right)} R^{i}\left[e_{-\alpha}\right] \otimes R_{i}\left[e_{\alpha^{\prime}}\right], \\
\Delta\left(e_{\beta}\right) R-R \Delta^{\prime}\left(e_{\beta}\right)= & t_{(\alpha)}^{\left(\alpha^{\prime}\right)}\left(R^{i}\left[e_{-\alpha}\right] \otimes e_{\beta} R_{i}\left[e_{\alpha^{\prime}}\right]\right. \\
& +e_{\beta} R^{i}\left[e_{-\alpha}\right] \otimes e^{\varphi(\beta, \cdot)} R_{i}\left[e_{\alpha^{\prime}}\right]-R^{i}\left[e_{-\alpha}\right] e_{\beta} \otimes R_{i}\left[e_{\alpha^{\prime}}\right] \\
& \left.-R^{i}\left[e_{-\alpha}\right] e^{\varphi(\beta, \cdot)} \otimes R_{i}\left[e_{\alpha^{\prime}}\right] e_{\beta}\right) .
\end{aligned}
$$

Terms 2 and 3 combine to $R^{i}\left[e_{\beta}, t^{\left(\alpha^{\prime}\right)}\right] \otimes R_{i}\left[e_{\alpha^{\prime}}\right]$, and the recursion relations (7.2) implies that the sum of all four terms equals zero. Actually this recursion 
relation, when summed over $n$, is nothing more than the statement $\Delta\left(e_{\beta}\right) R-R \Delta^{\prime}\left(e_{\beta}\right)=0$. It should be pointed out that the co-product was not known a priori; the Yang-Baxter relation gave us the recursion relation and this amounts to a determination of the co-product.

(d) The existence and uniqueness of the homomorphism $\mathscr{E}$ and the anti-homomorphism $S$ are obvious. We have to show that $\mathscr{E}$ satisfies the axioms

$$
(\mathscr{E} \times i d) \Delta=i d=(i d \times \mathscr{E}) \Delta,
$$

which is straightforward, and that

$$
m(i d \times S) \Delta=\epsilon=m(S \times i d) .
$$

Here $m$ indicates multiplication, $\mathscr{A} \otimes \mathscr{A} \rightarrow \mathscr{A}$. For example,

$$
m(i d \times S) \Delta\left(e_{\alpha}\right)=S\left(e_{\alpha}\right)+e_{\alpha} e^{-\varphi(\alpha, \cdot)}=0 .
$$

(e) Obvious, since $\mathscr{E}(I)=0$ and $S(I)=I$ by Proposition 7.2. Proposition 14.1 is proved.

We turn to the case of the deformed R-matrix of Section 13, all statements should be understood to hold to first order in the deformation parameter $\epsilon$. The maps $\Delta, \mathscr{E}$ and $S$ are as before and the deformed maps are $\Delta_{\epsilon}=\Delta+\epsilon \Delta_{1}$, $\mathscr{E}_{\epsilon}=\mathscr{E}+\epsilon \mathscr{E}_{1}, S_{\epsilon}=S+\epsilon S_{1}$.

Proposition 14.2. (a) There is a unique homomorphism $\Delta_{\epsilon}: \mathscr{A} \rightarrow \mathscr{A} \otimes \mathscr{A}$ such that

$$
\Delta_{1}(x)=\left[\Delta(x), K e_{-\rho} \otimes K e_{\sigma}\right], \quad x \in \mathscr{A} .
$$

(b) The projection of $\Delta_{\epsilon}$ to $\mathscr{A}^{\prime} \rightarrow \mathscr{A}^{\prime} \otimes \mathscr{A}^{\prime}$ is well defined. (c) Let $\Delta_{t}^{\prime}$ be the opposite coproduct on $\mathscr{A}^{\prime}=\mathscr{A} / I$, and $R_{\epsilon}=R+\epsilon R_{1}$ the $R$-matrix of Theorem 13.1, then $\Delta_{\epsilon} R_{\epsilon}=R_{\epsilon} \Delta_{\epsilon}^{\prime}$ (to first order in $\epsilon$ ). (d) The deformed counit and antipode of $\mathscr{A}$ are given by $\mathscr{E}_{1}=0$ and

$$
S_{1}(x)=\left[K e_{-\rho} e_{\sigma}, S(x)\right], \quad x \in \mathscr{A} .
$$

(e) The counit $\mathscr{E}_{\mathfrak{\epsilon}}$ and the antipode $S_{\mathfrak{\epsilon}}$ induce analogous structures on $\mathscr{A} / I$.

Proof. (a) By the Jacobi identity. (b) Obvious, for $\Delta_{1}(C)=[\Delta(C)$, $\left.K e_{-\rho} \otimes K e_{\sigma}\right] \in I \otimes \mathscr{A}+\mathscr{A} \otimes I$. (c) Completely straightforward. (d) We have $(\mathscr{E} \times i d) \Delta_{1}(x)=0$, whence $\mathscr{E}_{1}=0$, while

$$
m\left(i d \times S_{1}\right) \Delta\left(H_{a}\right)+m(i d \times S) \Delta_{1}\left(H_{a}\right)=0
$$


since

$$
\begin{aligned}
m\left(i d \times S_{1}\right) \Delta\left(H_{a}\right)=S_{1}\left(H_{a}\right)=\left[H_{a}, K e_{-\rho} e_{\sigma}\right], & \\
m(i d \times S) \Delta_{1}\left(H_{a}\right) & =m(i d \times S)\left(H_{a}(\sigma)-H_{a}(\rho) K e_{-\rho} \otimes K e_{\sigma}\right. \\
& =\left(H_{a}(\sigma)-H_{a}(\rho)\right) K e_{-\rho}\left(-e_{\sigma} e^{-\varphi(\sigma, \cdot)} K^{-1}\right) \\
& =-\left(H_{a}(\sigma)-H_{a}(\rho)\right) K e_{-\rho} e_{\sigma}=-\left[H_{a}, K e_{-\rho} e_{\sigma}\right]
\end{aligned}
$$

and

$$
m\left(i d \times S_{1}\right) \Delta\left(e_{\alpha}\right)+m(i d \times S) \Delta_{1}\left(e_{\alpha}\right)=0
$$

since

$$
\begin{aligned}
m\left(i d \times S_{1}\right) \Delta\left(e_{\alpha}\right) & =S_{1}\left(e_{\alpha}\right)+e_{\alpha} S_{1}\left(e^{\varphi(\alpha, \cdot)}\right) \\
& =S_{1}\left(e_{\alpha}\right)-e_{\alpha}\left[e^{-\varphi(\alpha, \cdot)}, K e_{-\rho} e_{\sigma}\right] \\
& =\left[e_{\alpha}, K e_{-\rho} e_{\sigma}\right] e^{-\varphi(\alpha, \cdot)} . \\
m(i d \times S) \Delta_{1}\left(e_{\alpha}\right) & =-\left[e_{\alpha}, K e_{-\rho} e_{\sigma}\right] e^{-\varphi(\alpha, \cdot)} .
\end{aligned}
$$

These last two results require some work.

(e) This is clear, since $\mathscr{E}_{1}=0$ and $S_{1}(I) \in I$. The proposition is proved.

\section{§15. Exact Deformations of Standard, Generalized Quantum Groups}

We return to the first order deformations described in Theorem 13.1. A deformation of this type, involving a single pair $(\rho, \sigma)$ for which (13.3) holds, is called an elementary deformation. We shall see that, to each elementary, first order deformation, there is an exact deformation (to all orders in $\epsilon$ ), that can be expressed in closed form. To first order in $\epsilon$, the problem being then linear, one obtains a more general space of deformations by adding the contributions of several such pairs,

$$
R_{1}=\sum_{(\sigma, \rho) \in[\tau]}\left(R f_{\sigma} \otimes f_{-\rho}-f_{-\rho} \otimes f_{\sigma} R\right) .
$$

Here the sum is over a subset $[\tau]$ of the pairs $(\sigma, \rho) ; \sigma \in \hat{\Gamma}_{1}, \rho \in \hat{\Gamma}_{2}$, where $\hat{\Gamma}_{1,2}$ are subsets of the set of positive generators, and

$$
f_{\sigma}:=e^{-\varphi(\sigma, \cdot)} e_{\sigma}, \quad f_{-\rho}:=e_{-\rho} e^{\varphi(\cdot \rho)}, \quad e^{\varphi(\sigma,)+\varphi(\cdot, \rho)}=1, \quad(\sigma, \rho) \in[\tau] .
$$

Not all such compounded, first order deformations lift to exact deformations. 
The deformed co-product was also calculated to first order in $\epsilon$, and the results suggest an approach to the exact deformations. The formula (13.4) for $R_{1}$, as well as the expression (14.4) for the first order deformation of the coproduct, both suggest that the deformation be formulated as a twist [D3], but of a type much more general than that proposed by Reshetikhin [R]. Additional support for this is found in the fact that the exact, elementary deformations mentioned above and given below (see "Examples") are also of this type. For the following result $\mathscr{A}^{\prime}$ is any coboundary Hopf algebra.

Theorem 15.1. Let $R$ be the R-matrix, $\Delta$ the coproduct, of a coboundary Hopf algebra $\mathscr{A}^{\prime}$, and $F \in \mathscr{A}^{\prime} \otimes \mathscr{A}^{\prime}$, invertible, such that

$$
\left(\left(1 \otimes \Delta_{21}\right) F\right) F_{12}=\left(\left(\Delta_{13} \otimes 1\right) F\right) F_{31} .
$$

Then

$$
\tilde{R}:=\left(F^{t}\right)^{-1} R F
$$

(a) satisfies the Yang-Baxter relation and (b) defines a Hopf algebra $\tilde{\mathscr{A}}$ with the same product and with co-product

$$
\tilde{\Delta}=\left(F^{t}\right)^{-1} \Delta F^{t}
$$

Proof. (a) We substitute (15.4) into the expression $\tilde{R}_{12} \tilde{R}_{13} \tilde{R}_{23}$. Then use (15.3) to express $F_{12}\left(F_{31}\right)^{-1}$ in terms of the co-products, and the intertwining property of $R\left(\Delta R=R \Delta^{\prime}\right)$ to shift the latter to the ends. The rest is obvious. (b) It is clear that $\tilde{\Delta}$ is an algebra homomorphism. We shall show that the twisted coproduct defined by $\tilde{\Delta}$ is co-associative:

$$
\begin{aligned}
\left(1 \otimes \tilde{\Delta}_{21}\right) \tilde{\Delta}(x) & =F_{12}^{-1}\left(1 \otimes \Delta_{21}\right) \tilde{\Delta}(x) F_{12} \\
& =F_{12}^{-1}\left(1 \otimes \Delta_{21}\left(F^{t}\right)^{-1}\right)\left(1 \otimes \Delta_{21} \Delta(x)\right)\left(1 \otimes \Delta_{21} F^{t}\right) F_{12}, \\
\left(\tilde{\Delta}_{13} \otimes 1\right) \tilde{\Delta}(x) & =F_{31}^{-1}\left(\Delta_{13} \otimes 1\left(F^{t}\right)^{-1}\right)\left(\Delta_{13} \otimes 1 \Delta(x)\right)\left(\Delta_{13} \otimes 1 F^{t}\right) F_{31} .
\end{aligned}
$$

Comparing the factors at either end one finds that they agree by virtue of (15.3). The result follows, in view of the co-associativity of $\Delta$. The theorem is proved.*

\footnotetext{
* The connection between Eq.(15.3) and co-associativity was pointed out to me by Masaki
} Kashiwara. The relation makes $F$ a cocycle in the sense of Gerstenhaber [G]. 
We return to our subject, with $R$ again denoting the standard R-matrix of the algebra $\mathscr{A}^{\prime}=\mathscr{A} / I$. We show first that interesting solutions of (15.3) exist. Then we do some preliminary calculations that help us make a general ansatz for $F$ in the form of a double expansion, $F=\Sigma \epsilon^{n m} F_{n}^{m}$, and finally we derive a recursion relation for $F_{n}^{m}$ that will allow us to calculate the classical limit.

Examples. An exact deformation of $R$, with the first order term $R_{1}$ as in (15.1) but with the sum reduced to a single term, is given by

$$
F=e_{q}^{-\ell f_{\sigma} \otimes f-\rho},
$$

with

$$
f_{\sigma}:=e^{-\varphi(\sigma \cdot)} e_{\sigma}, \quad f_{-\rho}:=e_{-\rho} e^{\varphi(\cdot, \rho)}
$$

The q-exponential is as follows: $q=e^{\varphi(\sigma . \rho)}, e_{q}^{A}:=\Sigma A^{n} /[n !]_{q},[n !]_{q}=[1]_{q} \cdots[n]_{q}$, $[n]_{q}=\left(q^{n}-1\right) /(q-1)$. Note that, if $A B=q B A$, then $e_{q}^{A} e_{q}^{B}=e_{q}^{(A+B)}$. Proposition 15.1 shows that an elementary twist $F$, of the simple form (15.6), can be combined in a naive way with another elementary twist $\tilde{F}$, of the same type but with $(\sigma, \rho)$ replaced by $\left(\sigma^{\prime}, \rho^{\prime}\right)$, only if $\tilde{\Delta}\left(f_{\sigma^{\prime}}\right), \tilde{\Delta}\left(f_{\rho^{\prime}}\right)$ reduce to $\Delta\left(f_{\sigma}^{\prime}\right), \Delta\left(\rho^{\prime}\right)$; that is, only when the four generators quommute* among themselves.

Notation. From now on it will be convenient to use the generators $f_{ \pm \alpha}$ defined in (15.7). The standard co-product then takes the form

$$
\Delta f_{\sigma}=K^{\sigma} \otimes f_{\sigma}+f_{\sigma} \otimes 1, \quad \Delta f_{-\rho}=1 \otimes f_{-\rho}+f_{-\rho} \otimes K_{\rho},
$$

with

$$
K_{\rho}:=e^{\varphi(\cdot, \rho)}, \quad K^{\sigma}:=e^{-\varphi(\sigma, \cdot)}
$$

The general case of compound deformations is much more complicated. The calculations are manageable only so long as $F$ can be constructed from elements of the type $f_{\sigma} \otimes f_{-\rho}$ only, with the factors in this order. A general result is Theorem 15.2 below. We need some preparation.

Proposition 15.1. Let $R_{\epsilon}$ be an exact deformation of the type

$$
R_{\epsilon}=\left(F^{t}\right)^{-1} R F, \quad F=\sum \epsilon^{n}\left(F_{n}+\cdots\right)
$$

\footnotetext{
* Two elements $x, y$ of an algebra quommute if there is $q$ in the field such that $x y-q y x=0$.
} 


$$
F_{n}=\sum_{(\sigma . \rho) \in[\tau]} F_{\left(\sigma^{\prime}\right)}^{\left(\sigma^{\prime}\right)} f_{\sigma_{1}} \cdots f_{\sigma_{n}} \otimes f_{-\rho_{1}^{\prime}} \cdots f_{-\rho_{n}^{\prime}},
$$

where $+\cdots$ stands for terms with less than $n$ factors. Let $\Gamma_{1}, \Gamma_{2}$ be the subalgebras of $\mathscr{A}^{\prime+}$ generated by $\hat{\Gamma}_{1}, \hat{\Gamma}_{2}$. Then we have: (a) There is an isomorphism $\tau: \Gamma_{1} \rightarrow \Gamma_{2}$, such that the set $[\tau]$ is the restriction of the graph of $\tau$ to $\hat{\Gamma}_{1}, \hat{\Gamma}_{2}$,

$$
[\tau]=\left\{\sigma, \rho \mid \sigma \in \hat{\Gamma}_{1}, \quad \rho=\tau \sigma \in \hat{\Gamma}_{2}\right\} .
$$

(b) The elements $F_{n}$ satisfy the recursion relations

$$
\left[F_{n}, f_{-\sigma} \otimes 1\right]=\left(K^{\sigma} \otimes f_{-\rho}\right) F_{n-1}-F_{n-1}\left(K_{\sigma} \otimes f_{-\rho}\right), \quad(\sigma, \rho) \in[\tau],
$$

as well as

$$
\left[1 \otimes f_{\rho}, F_{n}\right]=F_{n-1}\left(f_{\sigma} \otimes K^{\rho}\right)-\left(f_{\sigma} \otimes K_{\rho}\right) F_{n-1} .
$$

(c) These recursion relations have the unique solution

$$
F_{(\sigma)}^{\left(g^{\prime}\right)}=(-)^{n} t_{(\sigma)}^{\left(\sigma^{\prime}\right)}, \quad\left(\rho_{1}^{\prime}, \cdots, \rho_{n}^{\prime}\right)=\tau\left(\sigma_{1}^{\prime}, \cdots, \sigma_{n}^{\prime}\right),
$$

where the coefficients on the right are the same as in Eq.(2.5), except that $\varphi$ is replaced by $\varphi^{t}$.

Proof. We begin by offering some justification for the assumptions. In view of the form of $R_{1}$ it is expected that $R_{n}$ is a sum of products of factors of three types:

$$
e_{-\alpha} \otimes e_{\alpha}, \quad f_{-\rho} \otimes f_{\sigma}, \quad f_{\sigma} \otimes f_{-\rho}, \quad \sigma \in \hat{\Gamma}_{1}, \quad \rho \in \hat{\Gamma}_{2},
$$

with coefficients in $\mathscr{A}^{\prime} \otimes \mathscr{A}^{\prime}$. In $R_{n}$, we isolate the terms with the highest number of factors of the third type,

$$
X_{n}=\sum A_{\left(\sigma^{\prime}\right)}^{\left(\rho^{\prime}\right)}\left(e_{-\alpha_{1}} \cdots e_{-\alpha_{k}} \otimes e_{\alpha_{1}} \cdots e_{\alpha_{k}}\right) B_{\left(\sigma_{)}^{\prime}\right)}^{\left(\rho^{\prime}\right)}\left(f_{\sigma_{1}} \cdots f_{\sigma_{n}} \otimes f_{-\rho_{1}} \cdots f_{-\rho_{n}^{\prime}}\right) .
$$

We shall show that $R_{n}$ contains $X_{n} \neq 0$.

Let

$$
Y B_{\epsilon}:=R_{\epsilon 12} R_{\epsilon 13} R_{\epsilon 23}-R_{\epsilon 23} R_{\epsilon 13} R_{\epsilon 12} \in \mathscr{A}^{\prime} \otimes A^{\prime} \otimes A^{\prime} .
$$

All terms in $Y B_{\epsilon}$ of order $\epsilon^{n}$, that have $n$ factors of the third type in spaces 1,2 are contained in 


$$
P_{n}:=X_{n 12} R_{13} R_{23}-R_{23} R_{13} X_{n 12}
$$

For these terms to cancel among themselves $X_{n}$ must take the form

$$
X_{n}=R F_{n}, \quad F_{n}=F_{(\sigma)}^{\left(\rho^{\prime}\right)}\left(f_{\sigma_{1}} \cdots f_{\sigma_{n}} \otimes f_{-\rho_{1}^{\prime}} \cdots f_{-\rho_{n}^{\prime}}\right)
$$

The sum is over all pairs $(\sigma, \rho) \in[\tau]$ and all permutations $\left(\rho^{\prime}\right)$ of $(\rho)$.

Next, the recursion relation (15.10) follows easily from the Yang-Baxter relation (more precisely from an examination terms of low order in space 2), and (15.11) from a similar calculation.

We have $F_{0}=1$ and $F_{1}=\Sigma f_{\sigma} \otimes f_{-\rho}$. Taking $n=1$ in (15.10) or (15.11) one gets,

$$
\left[f_{\alpha}, f_{-\beta}\right]=\delta_{\alpha}^{\beta}\left(e^{\varphi(\cdot, \alpha)}-e^{-\varphi(\alpha, \cdot)}\right),
$$

which is confirmed by the definitions in (15.7) and the relation (2.4). When (15.10) is reduced to a recursion relation for the coefficients, then it turns out to agree (up to a sign and $\varphi \rightarrow \varphi^{t}$ ) with the recursion relations (2.14) for the coefficients $t_{(\sigma)}^{\left(\sigma^{\prime}\right)}$. The integrability of these relations is precisely the statement (a) of the theorem, as follows easily from the analysis of these recursion relations in $\S 5$. Finally, when (a) holds, then the relation (15.11) is equivalent to (15.10). The proposition is proved.

After these preliminary explorations we are able to formulate a general result.

Theorem 15.2. Let $\Gamma_{1}, \Gamma_{2}$ be subalgebras of $\mathscr{A}^{+}$, generated by subsets $\hat{\Gamma}_{1}, \hat{\Gamma}_{2}$ of the generators, and $\tau: \Gamma_{1} \rightarrow \Gamma_{2}$ an algebra isomorphism. Let $F \in \mathscr{A}^{\prime} \otimes \mathscr{A}^{\prime}$ be a formal series of the form

$$
F=1+\sum_{n=1}^{\infty} \epsilon^{n} F_{n}, \quad F_{n}=\sum F_{(\sigma)}^{(\rho)} f_{\sigma_{1}} \cdots f_{\sigma_{n}} \otimes f_{-\rho_{1}} \cdots f_{-\rho_{n}} .
$$

The second sum is here over all $\sigma_{i} \in \hat{\Gamma}_{1}, \rho_{i} \in \hat{\Gamma}_{2}$. (!) Note that $F_{n}$ is a power series in $\epsilon$. Suppose that $F$ satisfies (15.3), and that

$$
F_{0}=1, \quad F_{1}=-\sum_{\tau^{m} \sigma=\rho} \epsilon^{m-1}\left(f_{\sigma} \otimes f-\rho\right)
$$

then $F_{n}$ satisfies

$$
\left(1 \otimes K_{\rho} \partial_{\rho}\right) F_{n}+\sum_{\tau^{m} \sigma=\rho} \epsilon^{m}\left[1 \otimes f_{\sigma}, F_{n}\right]+\sum_{\tau^{m} \sigma=\rho} \epsilon^{m-1}\left(f_{\sigma} \otimes K^{\sigma}\right) F_{n-1}=0
$$


With $F_{0}$ and $F_{1}$ thus fixed, $F_{2}, F_{3}, \cdots$ are determined recursively and uniquely. (The operator $K_{\rho} \partial_{\rho}$ is the derivation that replaces $f_{-\rho}$ by $K_{\rho}$.)

Notation. The sums in (15.19-20), and similar sums to follow, should be understood to run over $\sigma \in \hat{\Gamma}_{1}$ and over all values of the positive integer $m$ such that $\tau^{m} \sigma$ is defined; that is, all values of $m$ such that $\tau^{m-1} \sigma \in \hat{\Gamma}_{1}$.

Proof. The exact form (15.19) of $F_{1}$ can be inferred directly from the Yang-Baxter relation. That Eq. (15.3) implies (15.20) is a simple calculation; one collects all terms that have exactly one generator in the second space. Let us verify that the recursion relation is satisfied for $n=1$ by (15.19). The second term is

$$
-\sum_{\tau^{m^{\prime}} \sigma^{\prime}=\rho^{\prime}} \epsilon^{m^{\prime}} \sum_{\tau^{m}{ }_{\sigma=\rho}=\epsilon^{m-1}} f_{\sigma^{\prime}} \otimes\left[f_{\sigma}, f_{-\rho^{\prime}}\right]
$$

The commutator is

$$
\left[f_{\sigma}, f_{-\rho^{\prime}}\right]=e^{\varphi\left(\cdot, \rho^{\prime}\right)}-e^{-\varphi\left(\rho^{\prime}, \cdot\right)}=e^{\varphi\left(\cdot, \tau^{m^{\prime}} \sigma^{\prime}\right)}-e^{\varphi\left(\cdot, \tau^{m^{\prime}+1} \sigma^{\prime}\right)} .
$$

The double sum reduces to $\Sigma_{\tau^{m} \sigma=\rho} \epsilon^{m-1} f_{\sigma} \otimes\left(K^{\sigma}-K_{\rho}\right)$ and (15.20) reduces to an identity. It remains to prove that $(15.20)$ has a unique solution. Consider first the case that $\hat{\Gamma}_{1} \cap \hat{\Gamma}_{2}$ is empty; then the second term in (15.20) vanishes and the third term reduces to the term $m=1$. The recursion relation then reduces to the same form as that which determines the coefficients of the standard R-matrix, which is known to be integrable. (In this case Proposition 15.1 is the complete solution of the problem, for there are no terms " $+\cdots$ " in (15.8).) In the general case, when $\hat{\Gamma}_{1} \cap \hat{\Gamma}_{2}$ can be nonempty, the second term in (15.20) makes the solution more difficult, but the existence of a solution can still be proved. To do this we expand $F_{n}$ as a power series in $\epsilon$, with constant term

$$
F_{n}^{1}=\sum F_{(\sigma)}^{\left(\tau \sigma^{\prime}\right)} f_{\sigma_{1}^{\prime}} \cdots f_{\sigma_{n}^{\prime}} \otimes f_{-\tau \sigma_{1}^{\prime}} \cdots f_{-\tau \sigma_{n}^{\prime}},
$$

and determine the coefficients recursively. The problem is therefore always the integrability of $K_{\rho} \partial_{\rho} X=Y, \rho \in \hat{\Gamma}_{2}$, with $Y \in \mathscr{A}^{\prime}$ given, and this we know to have a unique solution in $\mathscr{A}^{\prime}$, as already noted. The theorem is proved.

The converse, that the solution of (15.20) with $F_{0}=1$ and $F_{1}$ given by (15.19) satisfies (15.3) (and therefore gives a solution of the Yang-Baxter relation) 
was proved only in the special case that $\hat{\Gamma}_{1} \cap \hat{\Gamma}_{2}$ is empty. Further direct computation supports the idea that $R_{\epsilon}$ always has the form $\left(F^{t}\right)^{-1} R F^{t}$, with $F$ of the form assumed in (15.18). This is strong support for the belief that the solution of the recursion relation (15.20), which was proved to exist always, actually furnishes the solution to the problem of exact deformations in the general case. The results stated in Theorems 13.1 and 13.2 may also be considered as strong evidence. As we shall see, additional favorable evidence comes from an examination of the classical limit. To prepare for this we need

Proposition 15.2. Let

$$
F_{n}^{m}=\sum_{\rho=\tau^{m} \sigma} T_{(\sigma)}^{\left(\sigma^{\prime}\right)} f_{\sigma_{1}} \cdots f_{\sigma_{n}} \otimes f_{-\tau^{m} \sigma_{1}^{\prime}} \cdots f_{-\tau^{m} \sigma_{n}^{\prime}}, \quad F_{0}^{m}=1
$$

in which the sum extends over $\sigma_{i} \in \hat{\Gamma}_{1},\left(\sigma^{\prime}\right)$ a permutation of $(\sigma)$, and the coefficients $\vec{t}_{(\sigma)}^{\left(\sigma^{\prime}\right)}$ are the same as in (15.12). Then the unique solution of (15.20) is

$$
\begin{aligned}
& F_{n}=\sum_{\Sigma n_{i}=n} \epsilon^{n_{2}+2 n_{3}+\cdots} F_{n_{1}}^{1} F_{n_{2}}^{2} \cdots=F_{n}^{1}+\epsilon F_{n-1}^{1} F_{1}^{2}+\epsilon^{2}\left(F_{n-2}^{1} F_{2}^{2}+F_{n-1} F_{1}^{3}\right)+\cdots \\
& F=\sum \epsilon^{n} F_{n}=\sum \epsilon^{n_{1}+2 n_{2}+\cdots} F_{n_{1}}^{1} F_{n_{2}}^{2} \cdots=F^{1} F^{2} \cdots, \quad F^{m}=\sum \epsilon^{n m} F_{n}^{m}
\end{aligned}
$$

For a proof, see below.

\section{§16. Esoteric r-matrices}

a) Quantized Kac-Moody algebra of finite type.

Proposition 16.1. If $\mathscr{A}^{\prime}$ is a quantized Kac-Moody algebra of finite type, then $\hat{\Gamma}_{1}$ is a proper subset of the set of positive generators and $\tau^{m+1} \hat{\Gamma}_{1} \cap \hat{\Gamma}_{1}$ is a proper subset of $\tau^{m} \hat{\Gamma}_{1} \cap \hat{\Gamma}_{1}$.

Proof. Suppose that the statement is false. Then there is $f_{\sigma} \in \hat{\Gamma}_{1}$ such that $\tau^{m} f_{\sigma} \in \hat{\Gamma}_{1}$ for all $m$, and consequently $\tau^{k} f_{\sigma}=f_{\sigma}$ for some $k$. But the condition (15.2) on the parameters, in the classical limit, implies that

$$
\varphi\left(\tau^{m} \sigma, \cdot\right)+\varphi\left(\cdot, \tau^{m+1} \sigma\right)=0 .
$$

Summing over $m=0,1, \cdots, k-1$ we obtain

$$
\sum_{m}\left(\varphi+\varphi^{t}\right)\left(\tau^{m} \sigma\right)=0
$$


which contradicts the fact that the Killing form is non-degenerate.

In the classical limit

$$
R_{\epsilon}=1+\hbar r_{\epsilon}+o\left(\hbar^{2}\right) \quad r_{\epsilon}=r+\epsilon+o\left(\epsilon^{2}\right) .
$$

In the case of an exact elementary deformation $R_{\epsilon}$, the associated exact deformation $r_{t}$ of $r$ coincides with the first order,

$$
r_{\epsilon}=r+\epsilon r_{1} \text {. }
$$

Consider the general case of an exact deformation of $R$ of the form postulated in Theorem 15.2. Define $X_{\epsilon}$ by

$$
F=1+\hbar X_{\epsilon}+o\left(\hbar^{2}\right)
$$

so that

$$
r_{\epsilon}=r+X_{\epsilon}-X_{\epsilon}^{t}
$$

Notation. In this section the symbols $\Gamma_{1,2}$ stand for Lie algebras, the classical limits of the algebras so designated until now.

From the fact that the coefficients in the expansion (15.21) of $F$ are the same as the coefficients in the expansion (2.5) of the standard R-matrix, and the known classical limit of the standard R-matrix for a Kac-Moody algebra of finite type, we get without calculation that

$$
X_{\epsilon}=-\sum_{m} \sum_{E_{i} \in \Gamma_{1}} \epsilon^{n m} E_{i} \otimes E_{-\tau^{m}}
$$

in which $n$ is the height of $E_{i}$. The normalization is the same as in $\S \S 9-10$; more precisely it is fixed as follows. (a) The set $\left\{E_{i}\right\}$ includes the generators of $\Gamma_{1}$. (b) The statement (9.9).* Consequently,

$$
r_{\epsilon}=r-\sum_{m} \sum_{\substack{E_{i} \in \Gamma_{1}^{1} \\ E_{j}=\tau^{m} E_{i}}} \epsilon^{n m} E_{i} \wedge E_{-j}
$$

The sums are finite, by Proposition 16.1. A renormalization exists that reduces the numerical coefficients to unity $(\epsilon$ in $C$ ); the result is in complete agreement

* Condition (b) can be re-phrased as follows. Let $\Gamma_{1}^{-}$be the Lie algebra generated by $\left\{f_{-\sigma}\right\}$, $f_{\sigma} \in \hat{\Gamma}_{1}$ and $\Gamma$ the Lie algebra generated by $\left\{f_{ \pm \sigma}\right\}, f_{\sigma} \in \hat{\Gamma}_{1}$. Then $\Sigma_{E_{i} \in \Gamma_{1}} E_{i} \otimes E_{-i}$ is the projection on $\Gamma_{1} \otimes \Gamma^{-}$of a $\Gamma$-invariant element of $\Gamma \otimes \Gamma$. 
with [BD].

b) Deformations in the affine case. Let $\mathscr{A}^{\prime}$ be a quantized Kac-Moody algebra of affine type. Two cases should be distinguished. If the subsets $\hat{\Gamma}_{1,2}$ of positive roots do no include the imaginary root $e_{0}$, then the formula (16.6) applies without change, except that now $r$ is one of the standard affine r-matrices determined earlier, Eq.s (9.15), (10.5), (11.4) or (11.5). There is nothing more to be said about this case and we turn our attention to the other one.

What merits special attention is the possibility that the first order deformation (15.1) may include one of the following

$$
e_{0} \wedge e_{-\rho}=\mu\left(E_{-} \otimes e_{-\rho}\right)-\lambda\left(e_{-\rho} \otimes E_{-}\right),
$$

or

$$
e_{\sigma} \wedge e_{-0}=\lambda^{-1}\left(e_{\sigma} \otimes E_{+}\right)-\mu^{-1}\left(E_{+} \otimes e_{\sigma}\right)
$$

with

$$
\varphi(\cdot, \rho)+\varphi(0, \cdot)=0, \quad \text { resp. } \varphi(\cdot, 0)+\varphi(\sigma, \cdot)=0,
$$

which implies that $\rho \neq 0$, resp. $\sigma \neq 0$. A simple renormalization, that connects the principal picture to the homogeneous picture, brings (16.8) to the form

$$
e_{\sigma} \wedge e_{-0}=\sqrt{\mu / \lambda}\left(e_{\sigma} \otimes E_{+}\right)-\sqrt{\lambda / \mu}\left(E_{+} \otimes e_{\sigma}\right) .
$$

To deal with the general case of exact deformations it is useful to note the following

Proposition 16.2. If $\mathscr{A}^{\prime}$ is a quantized Kac-Moody of affine type, then either the statement about $\hat{\Gamma}_{1}$ in Proposition 16.1 continues to hold, or $\mathscr{A}^{\prime}$ is of type $A_{N-1}^{(1)}, \hat{\Gamma}_{1}$ consists of all the positive generators, and $\tau$ generates the cyclic group of order $N$.

Proof. Suppose there is $f_{\sigma} \in \hat{\Gamma}_{1}$ such that $\tau^{N} f_{\sigma}=f_{\sigma}$ for some $N$. Then the Killing form is degenerate. But it is known [K] that any subalgebra of a Kac-Moody algebra of affine type, obtained by removing one generator, is a Kac-Moody algebra of finite type. It follows that $\hat{\Gamma}_{1}$ contains all the positive generators and exactly one $\tau$ orbit. Then $\hat{\Gamma}_{1}=\hat{\Gamma}_{2}$ and $\tau$ lifts to an isomorphism of the Dynkin diagram, which implies the result.

In this section we exclude the exceptional case. This means that the classical 
limit of $\Gamma_{1}$ is a finite dimensional Lie algebra, so that (16.6) can be applied directly, since the sum is finite.

Alternatively, the classical limit can be found with the help of the recursion relation

$$
\left(1+K_{\rho} \partial_{\rho}\right) F_{n}^{m}=-\left(f_{\sigma} \otimes K_{\rho}\right) F_{n-1}^{m}, \quad \tau^{m} \sigma=\rho,
$$

or better, the equivalent relation

$$
\left[1 \otimes f_{\rho}, F_{n}^{m}\right]=-\left(\left(f_{\sigma} \otimes K_{\rho}\right) F_{n-1}^{m}-F_{n-1}^{m}\left(f_{\sigma} \otimes K^{\rho}\right)\right)
$$

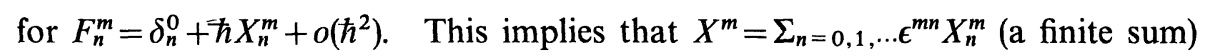
is the unique solution (of the form that appears in (16.5)) of

$$
\left[1 \otimes f_{\rho}+\epsilon^{m} f_{\sigma} \otimes 1, X^{m}\right]=\epsilon^{m} f_{\sigma} \otimes\left(\varphi+\varphi^{t}\right)(\rho), \quad \tau^{m} \sigma=\rho \in \hat{\Gamma}_{2} .
$$

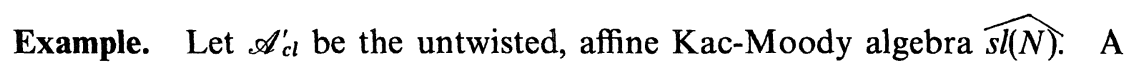
set of positive Serre generators is provided by the unit matrices $e_{i}=e_{i, i+1}$, $i=1, \cdots, N-1$. Set $e_{N}=e_{0}=\lambda e_{N 1}$. The "most esoteric" deformation (the one with the largest $\Gamma_{1}$ ) is defined as follows. Take $\Gamma_{1}$ to be generated by $e_{i}$, $i=1, \cdots, N-1$, and $\tau e_{i}=e_{i+1}, i=1, \cdots, N-1$. Then $X^{m}=\Sigma_{n} \epsilon^{n m} X_{n}^{m}$ with

$$
X_{n}^{m}=-\sum_{i+m+n \leq N} e_{i, i+n} \otimes e_{i+m+n, i+m}-\sum_{i+m+n=N+1} e_{i, i+n} \otimes \lambda^{-1} e_{1, i+m}
$$

and

$$
r_{\epsilon}=r+\left(\sum \epsilon^{n m} X_{n}^{m}-\text { transpose }\right)
$$

Taking $N=3$ one obtains

$$
r_{\epsilon}=r-\left(\epsilon e_{12} \otimes e_{32}+\epsilon^{2} e_{13} \otimes \lambda^{-1} e_{12}+\epsilon^{2} e_{12} \otimes \lambda^{-1} e_{13}-\text { transpose }\right)
$$

and the renormalization $e_{i j} \rightarrow \lambda^{\frac{j-i}{3}} e_{i j}$ gives the final result

$$
\begin{aligned}
r_{\epsilon}=r & -\epsilon\left\{\xi^{-1} e_{12} \otimes e_{32}+\xi^{-1} e_{23} \otimes e_{13}+\xi^{-2} e_{13} \otimes e_{12}\right\}-\epsilon^{2} \xi^{-1} e_{12} \otimes e_{13} \\
& +\epsilon\left\{\xi e_{32} \otimes e_{12}+\xi e_{13} \otimes e_{23}+\xi^{2} e_{12} \otimes e_{13}\right\}+\epsilon^{2} \xi e_{13} \otimes e_{12},
\end{aligned}
$$

with $\xi=(\lambda / \mu)^{1 / 3}$. The un-deformed piece is

$$
r=\varphi+\sum_{i<j} e_{i j} \otimes e_{j i}=\frac{1}{3}\left(\sum e_{i i} \otimes e_{i i}-e_{11} \otimes e_{22}-e_{22} \otimes e_{33}-e_{33} \otimes e_{11}\right)+\sum_{i<j} e_{i j} \otimes e_{j i},
$$


$\varphi$ being fixed by the relations (15.2). This is in agreement with [BD], after transposition and setting $\xi=e^{u / 3}, \epsilon=1$.

\section{§17. Universal Elliptic $\mathbf{R}$ and $\mathbf{r}$-matrices}

Here we consider the exceptional case (Proposition 16.2) in which $\hat{\Gamma}_{1}$ contains all the generators of $\mathscr{A}^{+}, \mathscr{A}^{\prime}$ is of type $A_{N-1}^{(1)}$ and $\tau^{N}=1$. $^{*}$ The expression (15.19) for $F_{1}$ can be justified as before and the sum is convergent if we interpret $\epsilon$ in $C$ and stipulate that

$$
|\epsilon|<1
$$

namely

$$
F_{1}=\frac{-1}{1-\epsilon^{N}} \sum_{m=1}^{N} \sum_{\sigma \in \Gamma_{1}} \epsilon^{m} f_{\sigma} \otimes f_{-\tau^{m} \sigma}
$$

Most, but not all, of the infinite sums that arise can be made meaningful in this way. In particular, (15.20) becomes

$$
\left(1-\epsilon^{N}\right)\left(1 \otimes K_{\rho} \partial_{\rho}\right) F_{n}+\sum_{m=1}^{N} \epsilon^{m}\left[1 \otimes f_{\tau}-m_{\rho}, F_{n}\right]+\sum_{m=1}^{N} \epsilon^{m-1}\left(f_{\tau}-m_{\rho} \otimes K^{\tau-m} \rho\right) F_{n-1} .
$$

We verify directly that it holds for $n=1$. The second term is

$$
\begin{aligned}
& \frac{-1}{1-\epsilon^{N}} \sum_{n=1}^{N} \sum_{m=1}^{N} \epsilon^{m+n} f_{\tau-m-n_{\rho}} \otimes\left(K_{\tau}-m_{\rho}-K_{\tau^{1-m} \rho}\right) \\
& =\frac{-1}{1-\epsilon^{N}} \sum_{M=1}^{N} \epsilon^{M} f_{\tau}-M_{\rho} \otimes\left(K^{\tau-M} \rho-K_{\rho}\right)\left(1-\epsilon^{N}\right) .
\end{aligned}
$$

The terms $K^{\tau^{-M}}, K_{\rho}$ comes from the ends of the summation while all the other terms cancel pairwise since $K^{\sigma}=K_{\tau \sigma}$.

The infinite product

$$
F=F^{1} F^{2} \ldots
$$

cannot be given anything more than a formal significance in the structural context but, as will be shown below, in a finite dimensional representation the

* The condition (15.2) can be satisfied on the loop algebra only, not on the full Kac-Moody algebra. 
question of convergence (with $\epsilon$ in $C$ ) is not difficult. We define $F^{m}$ by the (always uniquely integrable) relation (16.10),

$$
\left(1 \otimes K_{\rho} \partial_{\rho}\right) F^{m}=-\epsilon^{m}\left(f_{\tau-m_{\rho}} \otimes K_{\rho}\right) F^{m}, \quad F^{m}=1-\epsilon^{m} \sum_{\sigma} f_{\sigma} \otimes f_{-\tau^{m} \sigma}+o\left(\epsilon^{2 m}\right),
$$

or its equivalent

$$
\left[1 \otimes f_{\sigma}, F^{m}\right]=-\epsilon^{m}\left(\left(f_{\tau}-m_{\sigma} \otimes K_{\sigma}\right) F^{m}-F^{m}\left(f_{\tau}-m_{\sigma} \otimes K^{\sigma}\right)\right),
$$

with the same initial condition. We verify that, with this definition of $F^{m}$, (17.3) satisfies (17.2) or

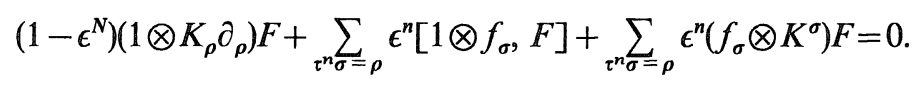

The range of the summation is $n=1,2, \cdots, N, \sigma \in \hat{\Gamma}_{1}$. One has

$$
\begin{aligned}
& \sum_{n} \epsilon^{n}\left[1 \otimes f_{-\tau^{-n} \rho}, F^{m} F^{m+1}\right]=-\sum_{n} \epsilon^{m+n} f_{\tau^{-m-n_{\rho}}} \otimes K_{\tau}-n_{\rho} F^{m} F^{m+1} \\
& +F^{m}\left\{\sum_{n} \epsilon^{m+n} f_{\tau^{-m-n} \rho} \otimes K^{\tau^{-n} \rho}-\sum_{n} \epsilon^{m+n+1} f_{\tau^{-m-n-1} \rho} \otimes K^{\tau-n-1} \rho\right\} F^{m+1}+\cdots
\end{aligned}
$$

In the second line everything cancels except for the first and the last terms, leaving

$$
-\sum_{n} \epsilon^{m+n}\left(f_{\tau-m-n_{\rho}} \otimes K_{\tau-n}\right) F^{m} F^{m+1}+\left(1-\epsilon^{N}\right) F^{m} \epsilon^{m+1}\left(f_{\tau-m-1} \otimes K_{\rho}\right) F^{m+1}+\cdots
$$

The total contribution of the commutator in (17.6) is thus

$$
-\sum_{n=1}^{N} \epsilon^{n+1}\left(f_{\tau}-n-1 \rho \otimes K_{\tau}-n_{\rho}\right) F+\left(1-\epsilon^{N}\right) \sum_{m=1}^{\infty} F^{1} \cdots F^{m} \epsilon^{m+1}\left(f_{\mathfrak{\tau}-m-1} \otimes K_{\rho}\right) F^{m+1} \cdots
$$

Adding the first term in (17.6) leaves us with

$$
-\sum_{n} \epsilon^{n+1}\left(f_{-\tau^{-n-1} \rho} \otimes K_{\tau^{-n} \rho}\right) F-\epsilon\left(1-\epsilon^{N}\right)\left(f_{-\tau^{-1} \rho} \otimes K_{\rho}\right) F=-\sum_{n} \epsilon^{n}\left(f_{-\tau^{-n} \rho} \otimes K^{\tau^{n} \rho}\right) F,
$$

which is cancelled by the last term.

In the classical limit $F^{m}=1+\hbar X^{m}+o\left(\hbar^{2}\right)$ and $X^{m}$ satisfies (16.12). We shall solve these relations in the case of the simplest affine Kac-Moody algebra. Set 


$$
\left[f_{1}, f_{-1}\right]=\left(\varphi+\varphi^{\dagger}\right)(1)=: H, \quad \tau f_{1}=f_{0}, \quad \tau f_{0}=f_{1},
$$

and

$$
H \otimes H+B^{m}\left(f_{1} \otimes f_{-1}+f_{0} \otimes f-0\right)+C^{m}\left(f_{1} \otimes f_{-0}+f_{0} \otimes f_{-1}\right)
$$

and impose (16.12). The result is, with $x=\lambda / \mu$,

$$
\begin{aligned}
A^{m} & =\frac{1}{2 \varphi(1,1)} \sum_{n=1}^{\infty}\left(-\epsilon^{2 n}\right)^{m} x^{-n}, \\
B^{2 m} & =\sum_{n=1}^{\infty}\left(\epsilon^{2 n-1}\right)^{2 m} x^{1-n}, \quad B^{2 m-1}=0, \\
C^{2 m-1} & =\sum_{n=1}^{\infty}\left(\epsilon^{2 n-1}\right)^{2 m-1} x^{1-n}, \quad C^{2 m}=0 .
\end{aligned}
$$

The deformed r-matrix is $r_{t}=r+X-X^{t}$, with

$$
\begin{aligned}
X=\sum_{n=1}^{\infty} X^{m}=\sum_{n} \frac{-\epsilon^{2 n}}{1+\epsilon^{2 n}} x^{-n} \frac{H \otimes H}{2 \varphi(1,1)} & +\sum_{n=1}^{\infty} \frac{\epsilon^{4 n-2}}{1-\epsilon^{4 n-2}} x^{1-n}\left(f_{1} \otimes f-1+f_{0} \otimes f_{-0}\right) \\
& +\sum_{n=1}^{\infty} \frac{\epsilon^{2 n-1}}{1-\epsilon^{4 n-2}} x^{1-n}\left(f_{1} \otimes f_{-0}+f_{0} \otimes f-1\right)
\end{aligned}
$$

Setting $\lambda / \mu=e^{2 \pi i u}$ one gets

$$
\begin{aligned}
(i / 2)\left(X-X^{t}\right)= & \sum_{n=1}^{\infty}\left\{\frac{-\epsilon^{2 n}}{1+\epsilon^{2 n}} \frac{H \otimes H}{2 \varphi(1,1)} \sin 2 n \pi u\right. \\
& +\frac{\epsilon^{4 n-2}}{1-\epsilon^{4 n-2}}\left(x f_{1} \otimes f_{-1}+\frac{1}{x} f_{-1} \otimes f_{1}\right) \sin (2 n-1) \pi u \\
& \left.+\frac{\epsilon^{2 n-1}}{1-\epsilon^{4 n-2}}\left(\sqrt{1 / \mu \lambda} f_{1} \otimes f_{1}+\sqrt{\mu \lambda} f_{-1} \otimes f_{-1}\right) \sin (2 n-1) \pi u\right\}
\end{aligned}
$$

The trigonometric r-matrix $(9.15)$ is

$$
\frac{i}{2}\left(\frac{1}{\tan \pi u} \frac{H \otimes H}{2 \varphi(1,1)}+\frac{1}{\sin \pi u}\left(e^{\pi i u} f_{1} \otimes f_{-1}+e^{-\pi i u} f_{-1} \otimes f_{1}\right)\right)
$$

Adding, one finds the series expansion of elliptic functions, and complete agreement with the elliptic r-matrices of [BD]. To transform to their notation replace

$$
f_{1} \rightarrow \sqrt{\lambda} e_{12}, \quad f_{-1} \rightarrow \sqrt{1 / \lambda} e_{21}
$$


Finally, we shall show that the expression for the Universal Elliptic R-matrix $R_{\epsilon}=\left(F^{t}\right)^{-1} R F, F=F^{1} F^{2} \ldots$ in terms of an infinite product is both meaningful and useable, by projecting on a finite dimensional represention. We limit ourselves to the fundamental representation of $s l(2)$. After rescaling of the generators as in (17.7), $F^{m}$ and $R_{\epsilon}$ take the form

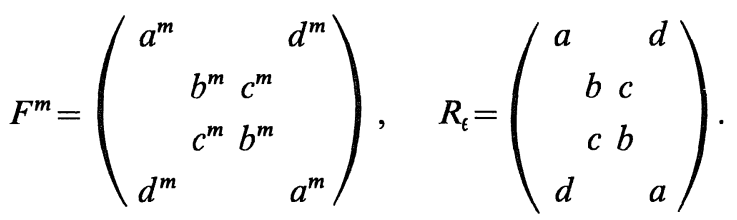

The matrix elements are completely determined by the recursion relation (17.5); namely for $m=1,2, \cdots, q^{2}=e^{\varphi(1,1)}=e^{2 \pi i \rho}$,

$$
\begin{gathered}
a^{2 m-1}=1-\epsilon^{4 m-2} \frac{1}{x}, b^{2 m-1}=1-\epsilon^{4 m-2} \frac{q^{2}}{x}, c^{2 m-1}=0, d^{2 m-1}=\epsilon^{2 m-1}\left(\frac{1}{q}-q\right) \sqrt{\frac{1}{x}} \\
a^{2 m}=1-\epsilon^{4 m} \frac{q^{2}}{x}, b^{2 m}=1-\epsilon^{4 m} \frac{1}{x}, c^{2 m}=\epsilon^{2 m} \sqrt{\frac{1}{x}}\left(\frac{1}{q}-q\right), d^{2 m}=0
\end{gathered}
$$

and

$$
a+d: a-d: b+c: b-c=\frac{d n(u+\rho)}{d n(u-\rho)}: 1: \frac{c n(u+\rho)}{\operatorname{cn}(u-\rho)}: \frac{\operatorname{sn}(u+\rho)}{\operatorname{sn}(u-\rho)} .
$$

A modular transformation brings this into perfect agreement with Baxter [B].

\section{Acknowledgements}

I am indebted to Moshe Flato for a detailed and constructive criticism of the original manuscript, and Masaski Kashiwara and Tetsuji Miwa for fruitful discussions. This work was completed at the Research Institute for Mathematical Sciences, Kyoto University. I thank the director, Professor Huzihiro Araki, for making the pleasant and productive stay in Kyoto possible, and for friendly hospitality during my visit. I thank the staff of the International Office of the Institute for many services, and the Japanese Ministry of Education, Science, Sports and Culture for financial support. 


\section{References}

[B] Baxter, R. J., Partition Function of the eight-Vertex Model, Ann. Physics, 70 (1972), 193-228.

[BD] Belavin, A. A. and Drinfeld, V. G., Triangle Equation and Simple Lie Algebras, Sov. Sci. Rev. Math., 4 (1984), 93-165.

[BP] Bidegain, F. and Pinczon, G., Quantization of Poisson Lie groups and applications, Dijon preprint 1995.

[BFGP] Bonneau, P., Flato, M., Gerstenhaber, M. and Pinczon, G., The hidden group structure of quantum groups: strong duality, rigidity and preferred deformations, Comm. Math. Phys., 161 (1994), 125-156.

[C] Chevalley, C., Sur la classification des algebres de Lie simples et de leur representations, C.R., 227 (1948), 1136-1138.

[CG] Cremmer, E. and Gervais, J.-L., The Quantum Group Structure Associated with Non-Linearly Extended Virasoro Algebras, Comm. Math. Phys., 134 (1990), 619-632.

[D1] Drinfeld, V. G., Quantum Groups, in Proceedings of the International Congress of Mathematicians, Berkeley, A. M. Gleason, ed. (American Mathematical Society, Providence 1987.)

[D2] - On some unsolved problems in quantum group theory, in Quantum Groups, P. P. Kulish, ed. Proceedings of the Workshop held in Euler International Mathematical Institute, Leningrad, Fall 1990.

[D3] — Quasi Hopf Algebras, Leningrad Math. J., 1 (1990), 1419-1457.

[EK] Etingof, P. and Kazhdan, D., Quantization of Poisson algebraic groups and Poisson homogeneous spaces, Harvard preprint, Oct. 20, 1955.

[FR] Frenkel, I. B. and Reshetikhin, N. Yu., Quantum Affine Algebras and Holonomic Difference Equations, Comm. Math. Phys., 146 (1992), 1-60.

[F] Frønsdal, C., Cohomology and Quantum Groups, in Proceedings of the Karpacz, Winter School, February 1994.

[FG1] Frønsdal, C. and Galindo, A., The Universal T-Matrix, Contemp. Math., 175 (1994), 73-88.

[FG2] , Deformations of Multiparameter Quantum $g l(N)$, Lett. Math. Phys., 34 (1995), 25-36.

[GK] Gabber, O. and Kac, V. G., On defining relations of certain infinite dimensional Lie algebras, Bull. Amer. Math. Soc., 5 (1980), 185-189.

[G] Gerstenhaber, M., The cohomology structure of an associative ring, Ann. Math., 78 (1963), 267-288.

[J] Jimbo, M., Quantum $R$ Matrix for the Generalized Toda System, Comm. Math. Phys., 102 (1986), 537-547.

[K] Kac, V. G., Infinite Dimensional Lie Algebras, Cambridge University Press 1990.

[KR] Kirillov, A. N. and Reshetikhin, N. Yu., q-Weyl group and a multiplicative formula for the universal R-matrix, Comm. Math. Phys., 134 (1991), 421-431.

[LS] Levendorski, S. Z. and Soibelman, Y. S., Some applications of quantum Weyl group I. The multiplicative formula for universal R-matrix for simple Lie algebras, J. Geom. Phys., 7 (1991), 1-14.

[Ma] Manin, Yu. I., Topics in noncommutative geometry, Princeton University Press, Princeton NJ 1991.

[Mo] Moody, R. V., A new class of Lie algebras, J. Algebra, 10 (1968), 211-230.

[R] Reshetikhin, N. Yu., Multiparameter Quantum Groups and Twisted Quasitriangular Hopf Algebras, Lett. Math. Phys., 20 (1990), 331-335.

[Ro] Rosso, M., An analogue of P.B.W. theorem and the universal R-matrix for $U_{h} s l(N)$, Comm. Math. Phys., 124 (1989), 307-319.

[Sc] Schirrmacher, A., The multiparametric deformation of $G L(n)$ and the covariant 
differential calculus on the quantum vector space, Z. Phys. C, 50 (1991), 321-327.

[Su] Sudbery, A., Fractional integral and differential equations for a class of Lévy-type probability densities, J. Phys. A, 23 (1990), L697-L700.

[T] Tanisaki, T., Killing forms, Harish-Chandra isomorphisms, and universal R-matrices for quantum algebras, Int. J. Mod. Phys. A, 7 Suppl. 1B (1992), 941-961.

[TV] Truini, P. and Varadarajan, V.S., Quantization of Reductive Lie Algebras, Rev. Math. Phys., 5 (1993), 363-415; Universal Deformations of Reductive Lie Algebras, Lett. Math. Phys., 26 (1992), 53-65. 
